Document downloaded from:

http://hdl.handle.net/10251/141448

This paper must be cited as:

Gao, P.; Climente Alarcón, A.; Sánchez-Dehesa Moreno-Cid, J.; Wu, L. (2019). Singlephase metamaterial plates for broadband vibration suppression at low frequencies. Journal of Sound and Vibration. 444:108-126. https://doi.org/10.1016/j.jsv.2018.12.022

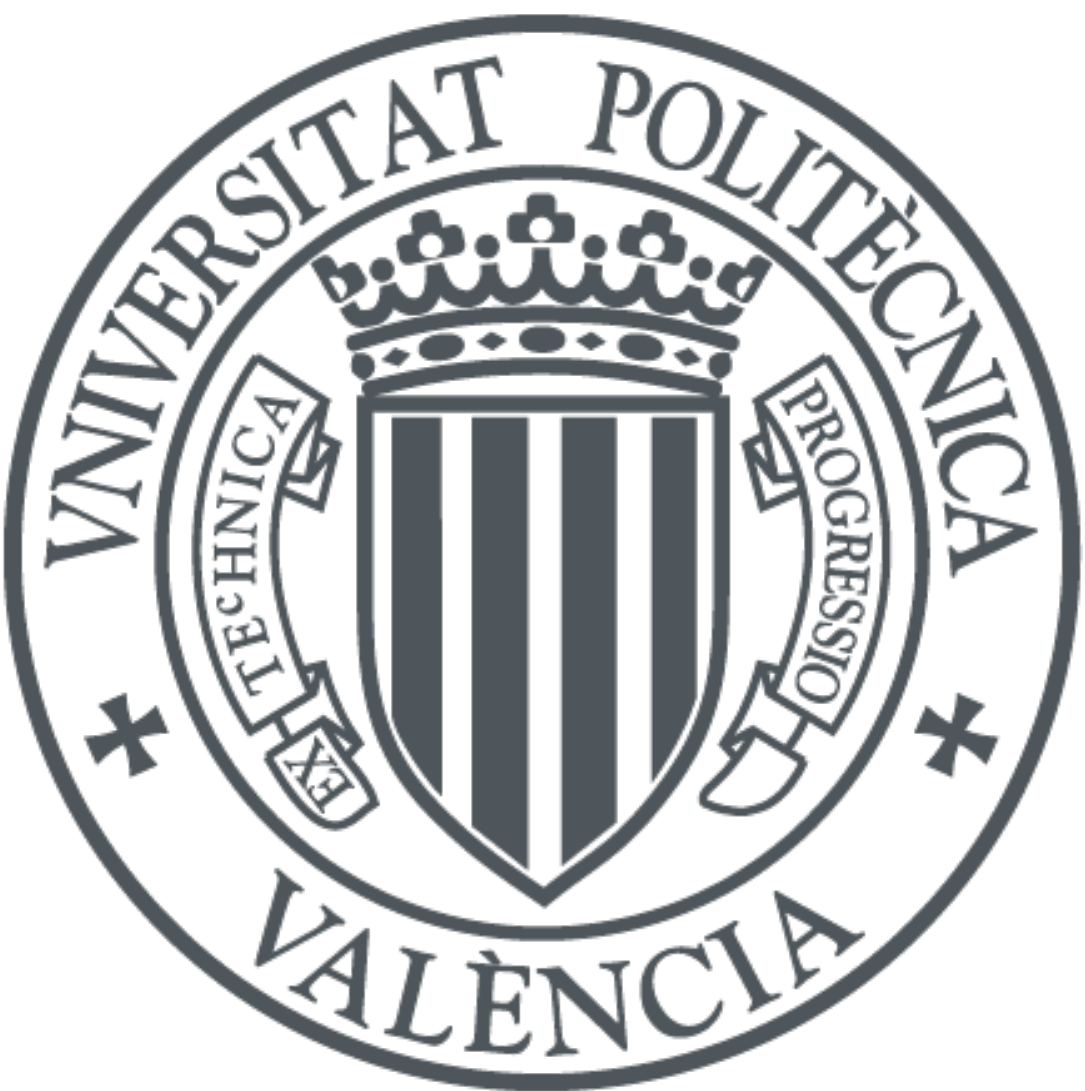

The final publication is available at

https://doi.org/10.1016/j.jsv.2018.12.022

Copyright Elsevier

Additional Information 


\title{
Single-phase metamaterial plates for broadband vibration suppression at low frequencies
}

\author{
Penglin $\mathrm{Gao}^{\mathrm{a}, \mathrm{b}}$, Alfonso Climente ${ }^{\mathrm{b}}$, José Sánchez-Dehesa ${ }^{\mathrm{b}, *}$, Linzhi $\mathrm{Wu}^{\mathrm{a}, \mathrm{c}, * *}$ \\ ${ }^{a}$ Center for Composite Materials and Structures, Harbin Institute of Technology, Harbin \\ 150001, China. \\ ${ }^{b}$ Wave Phenomena Group, Department of Electronic Engineering, Universitat Politècnica \\ de València, Camino de vera s.n. (Building 7F), ES-46022 Valencia, Spain. \\ ${ }^{c}$ Key Laboratory of Advanced Ship Materials and Mechanics, College of Aerospace and Civil \\ Engineering, Harbin Engineering University, Harbin 150001, China.
}

\begin{abstract}
By studying platonic crystals based on lattices of cavities containing $N$-beam resonators, we conclude that crystals made of 1-beam resonators easily produce low-frequency omnidirectional bandgaps. Based on this favorable property, hardly obtained for resonant cavities containing a higher number of beams $N \geq 2$, we have designed single-phase metamaterial plates for the suppression of low frequency flexural waves in a broad range of frequencies. These metamaterials are obtained by using resonant cavities containing a multiple number $M$ of identical 1-beam resonators uniformly distributed in the cavity. Square lattices of this type of resonators have been studied by using the impedance matrix approach and the multiple scattering method. This semi-analytical method has been employed to show the existence of complete bandgaps whose width can be optimized by increasing $M$. For the case $M=4$, the largest number of resonators studied here, three complete bandgaps separated by two narrow passbands appear in the band structure. The formation of these complete bandgaps originates from the dynamic interaction between different local resonators as well as their interaction with the propagating waves in the host plate. By using composite structures consisting of platonic crystal slabs with complementary bandgaps, these separated bandgaps easily merge into a broadband wave attenuation region. The normalized width, defined as the percentage of the bandwidth to its central frequency, reaches $95.3 \%$, representing an enhancement of about one order of magnitude compared with the absolute bandwidth obtained for the case of a single 1-beam resonator in the cavity. It is shown that the gaps can be easily tuned to lower frequencies by changing the geometrical parameters, such as the length of the beam, the radius and thickness of the smaller circular plate. Since the metamaterial is made of a single-phase material without attaching
\end{abstract}

\footnotetext{
${ }^{*}$ Corresponding author.

${ }^{* *}$ Corresponding author.

Email addresses: jsdehesa@upv.es (José Sánchez-Dehesa), wlz@hit.edu.cn (Linzhi Wu)
} 
heavy masses, the work reported here provides a simple approach to construct low-cost structures with potential applications in aeronautic and astronautic industries for broadband vibration suppression at low frequencies.

Keywords: Flexural waves in thin plates, Platonic crystals, Metamaterial plates, Vibration suppression in plates, Resonant bandgaps

\section{Introduction}

In the past decades, sonic/phononic crystals have been extensively studied owing to the rich physics associated to the propagation of acoustic/elastic waves propagating in fluids/solids with periodically arranged scatterers $[1,2]$. There 5 are many options for the scatterers and generally they contain holes, inclusions, pillars and a variety of well-designed resonators [3]. The lattices of resonators are termed as metamaterials because they behave like dynamic homogeneous materials within the long wavelength limit [4-6]. Owing to the out-of-phase response induced by the locally embedded resonances, the retrieved effective 10 parameters, such as density, bulk and shear moduli, may achieve negative values within the resonant bands $[4,7,8]$. The waves propagating in the metamaterials show many intriguing phenomena: low-frequency bandgaps [9], negative refraction [10], super-resolution imaging [11], and directional propagation [12], just to name a few. These anomalies not only enrich our understanding of wave

15 physics but also show a bright future in shielding, imaging and lensing.

Fueled by the seminal idea proposed by Liu et al. [9], various kinds of resonant structures were designed $[13,14]$ to create acoustic/elastic crystals with low-frequency bandgaps, not attainable by traditional scatterers because the Bragg-type bandgaps fall into frequency regions where the wavelength is comparable with the lattice constant. For waves propagating in solids, the magnitude of wavelength can be as large as several meters, and thus barriers for sound and vibration shielding cannot be fabricated in reasonable size unless employing local resonators. The excellent tunability endows metamaterials significant advantages in shielding low-frequency waves [15-18]. For airborne acoustics waves,

25 stacked panels composed of membrane-mass resonant units were demonstrated with an astonishing sound transmission loss (about $40 \mathrm{~dB}$ ) over a broad range from $50 \mathrm{~Hz}$ to $1000 \mathrm{~Hz}$ [15]. This achievement is extremely challenging for the mass-law-controlled sound barriers.

Our interest here is centered on reducing the low-frequency vibrations in

30 thin plates. The motivation originates from the basic reason that vibration problem needs to be solved urgently because of the bottleneck encountered in precision machining [19]. Periodic structures in thin plates are named as platonic crystals [20], and metamaterial plates based on them are likely to be one of the answers for the tough problem of vibration attenuation at low frequencies. Thus, 35 imitating the strategy proposed by Liu and coworkers [9], platonic crystals with coated composite structures were naturally introduced in the early works to open low-frequency bandgaps [21, 22]. Subsequently, numerous works focused 
their interest on attached resonators vibrating in the out-of-plane direction, typically including spring-masses [23-27], stubbed pillars [28-31] and beam-like 40 resonators [32].

The previously mentioned studies suffer from a drawback in practical fabrication on account of the high complexity and poor reliability of the attached structures, albeit the remarkable achievements in manipulating subwavelength waves. The reasons include three main parts. First, the resonant unit cell is 4 usually comprised of different constituent materials with highly contrasting material properties, making them hardly to be glued together steadily for a long time. Second, in most cases, heavy masses have to be attached on both sides of plates for the purpose of lowering bandgaps. Third, extra space is needed for the arrangement of attached resonators, which might be impracticable under ${ }_{50}$ some special conditions. The problem manifests further if one wants to broaden resonant bandgaps, and usually it means more complicated platonic structures have to be introduced [33, 34]. We notice that the nonlinear chaotic mechanism [35] and the dual mechanisms of shear stiffening and rotation softening [36] were applied to realize ultra-low and ultra-broad bandgaps. They present

55 elegant physical models for the understanding of wave physics, however, there is still a lot of work ahead due to the difficulties in practical implementation.

Single-phase metamaterial plates provide a more practical approach and, generally, they consist of holes with different inner structures easily to be fabricated by cutting machines. The cutting techniques bring favorable benefits in

60 the aspects of weight reduction and cost control. The aspect has attracted considerable interest in the community in recent years $[6,12,14,37-45]$. The previous works reported interesting applications in superlensing [39, 40], focusing [6, 44], directional wave guiding [12] and vibration shielding [37, 38, 41, 42, 45]. Among these works, most of them were performed within the framework of the finite element method because of the structural complexity. However, if the resonant microstructure is specially designed with simple geometries like rectangular beams and circular plates, such as the $N$-beam resonators proposed in Ref. [46], the theoretical study of the corresponding platonic crystals can be easily performed by combining the impedance matrix method with the mul70 tiple scattering method [44]. In most cases, the numerical computing based on the self-developed modeling is much more efficient than the finite-elementbased software when dealing with multiple scattering systems because the latter requires a huge number of elements to ensure accuracy.

This work presents the design of a metamaterial structure for the attenuation 75 of flexural waves at low frequencies. The design results from a comprehensive study of the properties of platonic crystals with resonant structures made of $\mathrm{N}$ beam resonators, which guides our attention to a solution based on a resonant cavity made of a multiple number $M$ of 1-beam resonators uniformly distributed in the cavity. Although previous works demonstrated that the number of 80 beams has a significant influence on the band structures [12, 39, 44, 45], only cavities with 1-beam $[39,45]$ and 2-beam $[12,44]$ resonators have been previously analyzed. Our present work studies cavities containing up to 4-beams and shows that cavity lattices made of 1-beam resonators are preferred since they 
easily provide complete bandgaps at low frequencies. Based on this finding, we have conceived resonant cavities with multiple 1-beam resonators providing simultaneously low-frequency broad bandgaps and excellent structural reliability. The resulting optimized metamaterial consists of a lattice of cavities containing four identical 1-beam resonators. Further support of this metamaterial plate has been provided by numerical simulations showing that a crystal slab made

90 of these cavities produces a total suppression of low-frequency flexural waves. We have concluded that these metamaterials can be used as light-weight and low-cost useful solutions in aeronautic and astronautic industries where the suppression of low-frequency vibration is a must.

The paper is organized as follows. After this introduction, Sec. 2 gives a brief description of the model employed to obtain the band structure of a square lattice of resonant cavities containing multiple $M$ number of 1-beam resonators uniformly distributed in the cavities. Next, in Sec. 3, we study the band structures of cavities containing multiple 1-beam resonators and compare them with the ones obtained when the resonant cavities contain $N$-beam resonators.

100 As a result of the comparison, we conclude that a resonant cavity containing four 1-beam resonators uniformly distributed is a better option to create broad bandgaps at low-frequencies. This result is further supported by analyzing the transmission properties of a metamaterial slab made of this type of cavities. Finally, the work is summarized in Sec. 4 with an outlook of application in

105 vibration suppression. Some useful but tedious developments are given in the Appendices.

\section{Modeling of resonant cavities containing $M$ 1-beam resonators}

In a previous work [44], the multiple scattering approach was applied to develop a semi-analytical model allowing the calculation of the band structure 110 of flexural waves propagating in a thin plate containing a lattice of resonant cavities. The cavities consist of $N$-beam resonators, whose corresponding Tmatrix was derived by applying the impedance matrix method [46]. In this section, we develop a similar procedure but now applied to the case of cavities containing a certain number $M$ of 1-beam resonators. As shown below, these

115 type of structures can exhibit complete bandgaps that are tunable in frequency by changing the geometrical parameters of the resonators. For the sake of comparison, Fig. 1 shows schematic diagrams of the two kinds of resonant cavities under study. On the one hand, Fig. 1(a) depicts the case of a cavity with a 2-beam resonator, which consists of an inner plate centered in the cavity and ${ }_{120}$ connected to the background plate by 2 rectangular beams with equal lengths $\ell=R_{2}-R_{1}$, where $R_{1}$ and $R_{2}$ are the radii of the smaller plate and cavity, respectively. On the other hand, Fig. 1(b) presents, as an example, the case of two $(M=2)$ 1-beam resonators inside a circular cavity with same radius $R_{2}$. In this work, we assume that all the resonators are uniformly distributed with 125 eccentric distance $e$. The length of the single beams is $\ell=r_{2}-r_{1}$, where $r_{1}$ and $r_{2}$ represent the radius of the smaller plates and the length of the segment $O_{n} \Psi_{n, 2}$, respectively. In both cases, all the beams are of equal width $b$, and $\theta_{n}$ 

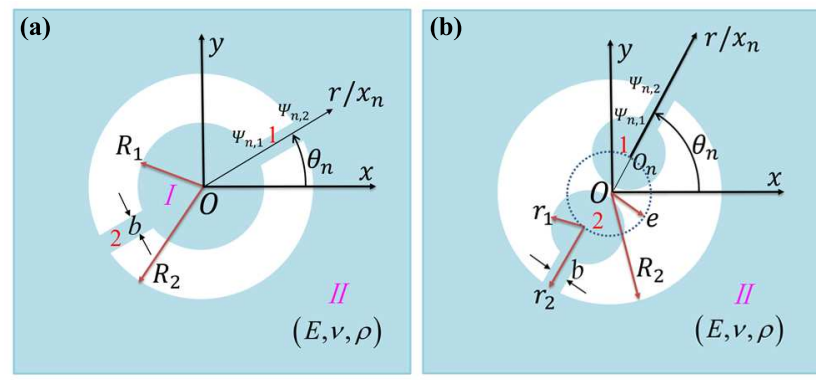

Fig. 1. (Color online) Schematic diagrams of the two kinds of resonant cavities under study. (a) Resonant cavity containing a $N$-beam resonator with $N=2$. The cavity consists of a smaller plate centered at the cavity and connected to the background plate with a number of $N=2$ rectangular beams. (b) Scheme corresponding to a resonant cavity containing $M$ 1-beam resonators, with $M=$ 2. Now, the cavity contains two 1-beam resonators uniformly distributed inside it. In both structures, the radius of the circular cavity is $R_{2}$, the width of all the beams is $b$, and $\theta_{n}$ defines the inclination angle of the $n$th beam which links the inner plate and background plate at anchor points $\Psi_{n, 1}$ and $\Psi_{n, 2}$, respectively. For the general case of a number $M$ of 1-beam resonators, all the resonators are radially structured with eccentric distance $e$. The radii of the inner disks are equal to $r_{1}$, different to $R_{1}$, the radius of the central disk of the $N$-beam resonators.

defines the inclination angle of the $n$th beam which links the inner plate and background plate at anchor points $\Psi_{n, 1}$ and $\Psi_{n, 2}$, respectively. As depicted in Fig. 1(b), the origins of the Cartesian coordinates $O(x, y)$ and polar coordinates $O(R, \Theta)$ are chosen at the center of the cavity. For convenience, the $n$th smaller plate is described in a local frame $O_{n}(r, \theta)$, and the corresponding beam is expressed in a local system $O_{n} x_{n}$ defined along the axis of the beam.

According to Kirchhoff-Love plate theory [47], the flexural waves propagating in thin plates are governed by the following equation of motion:

$$
\nabla^{4} W-k^{4} W=0
$$

where $k=\left(\rho h \omega^{2} / D\right)^{1 / 4}$ is the wave number, $\omega$ is the angular frequency, $h$ 135 denotes the plate thickness, and $D=E h^{3} / 12\left(1-\nu^{2}\right)$ is the rigidity with $E$, $\nu$ and $\rho$ being the Young's modulus, Poisson's ratio and mass density of the plate, respectively. The time harmonic factor $\exp (-\mathrm{i} \omega t)$ is implicit in all the formulation but will be omitted throughout the paper for simplicity.

In polar coordinates, the out-of-plane displacements in the background plate, $W(R, \Theta)$, and in the $n$th circular plate, $W^{n}(r, \theta)$, can be expressed as $[44,46]$

$$
W=\sum_{q=-\infty}^{\infty}\left[\mathbf{F}_{q}^{J I}\left(k_{p} R, \Theta\right) \mathbf{A}_{q}+\mathbf{F}_{q}^{H K}\left(k_{p} R, \Theta\right) \mathbf{B}_{q}\right]
$$




$$
W^{n}=\sum_{q=-\infty}^{\infty} \mathbf{F}_{q}^{J I}\left(k_{i} r, \theta\right)\left(\mathbf{C}_{n}\right)_{q}, \quad n \in[1, M]
$$

where $J, H, I$ and $K$ are the Bessel functions, and the matrix $\mathbf{F}_{q}^{\Upsilon \Phi}(\xi, \eta)=$ ${ }_{140}\left[\Upsilon_{q}(\xi) \Phi_{q}(\xi)\right] \mathrm{e}^{\mathrm{i} q \eta}$ is defined for simplicity. The column matrices $\mathbf{A}_{q}=\left[A_{q}^{J} A_{q}^{I}\right]^{t}$, $\mathbf{B}_{q}=\left[B_{q}^{H} B_{q}^{K}\right]^{t}$, and $\left(\mathbf{C}_{n}\right)_{q}=\left[C_{q}^{J} C_{q}^{I}\right]^{t}$ contain the expansion coefficients for the incoming, scattered and internal waves in the $n$th plate, respectively. The series summations are truncated in numerical simulations and run from $-N_{q}$ to $N_{q}$. The truncation order is set to $N_{q}=18$ for the calculations throughout 145 the paper. Moreover, $k_{i}$ and $k_{p}$ are wave numbers in the inner and background plates, respectively.

It is assumed that the structures depicted in Fig. 1 are fabricated by standard cutting techniques applied to a homogeneous plate and, consequently, within this single-phase metamaterial, $k_{i}$ equals to $k_{p}$ because all parts are made of the same material. However, this statement is not true for the case in which we need to increase the thickness of the circular plates $t$; a strategy that is adopted to adjust the fundamental resonance. This can be achieved in a practical way by pasting disks onto the circular plates (region I) with the same materials.

Regarding the short bridges connecting to the circular plates, they are considered as Euler-Bernoulli beams. Then, for one particular beam, its transverse displacement $V^{n}\left(x_{n}\right)$ is controlled by the following equation of motion [47]:

$$
\frac{\partial^{4} V^{n}}{\partial x_{n}^{4}}-k_{b}^{4} V^{n}=0, \quad n \in[1, M]
$$

where $k_{b}=\left(m \omega^{2} / E I\right)^{1 / 4}$ is the wave number in the beam with $I=b h^{3} / 12$ being the second moment of area and $m=\rho b h$ being the mass per unit length. Then, the solution within the beam can be expressed as [46]

$$
V^{n}\left(x_{n}\right)=D_{1}^{n} \mathrm{e}^{\mathrm{i} k_{b} x_{n}}+D_{2}^{n} \mathrm{e}^{-\mathrm{i} k_{b} x_{n}}+D_{3}^{n} \mathrm{e}^{k_{b} x_{n}}+D_{4}^{n} \mathrm{e}^{-k_{b} x_{n}} .
$$

The wave solutions expressed in Eqs. (2), (3) and (5) are related through the continuity conditions at the anchor points $\Psi_{n, 1}$ and $\Psi_{n, 2}$. Their expressions are

$$
\begin{gathered}
W^{n}\left(r_{1}, \theta_{n}\right)=V^{n}\left(r_{1}\right) \quad \text { and } \quad W\left(R_{2}, \theta_{n}\right)=V^{n}\left(r_{2}\right), \\
\left.\frac{\partial W^{n}}{\partial r}\right|_{\left(r_{1}, \theta_{n}\right)}=\left.\frac{\partial V^{n}}{\partial x_{n}}\right|_{r_{1}} \text { and }\left.\quad \frac{\partial W}{\partial R}\right|_{\left(R_{2}, \theta_{n}\right)}=\left.\frac{\partial V^{n}}{\partial x_{n}}\right|_{r_{2}}, \\
\left.M_{r}^{n}\right|_{\left(r_{1}, \theta_{n}\right)}=\left.\frac{M^{n}}{r_{1}}\right|_{r_{1}} \text { and }\left.\quad M_{R}\right|_{\left(R_{2}, \theta_{n}\right)}=\left.\frac{M^{n}}{R_{2}}\right|_{r_{2}}, \\
\left.V_{r}^{n}\right|_{\left(r_{1}, \theta_{n}\right)}=\left.\frac{Q^{n}}{r_{1}}\right|_{r_{1}} \text { and }\left.\quad V_{R}\right|_{\left(R_{2}, \theta_{n}\right)}=\left.\frac{Q^{n}}{R_{2}}\right|_{r_{2}},
\end{gathered}
$$


The coefficients of the incoming waves, $\mathbf{A}_{q}$, and scattered waves by the resonant cavity, $\mathbf{B}_{q}$, are related through the T-matrix as follows:

$$
\mathbf{B}_{q}=\sum_{s=-\infty}^{\infty} \mathbf{T}_{q s} \mathbf{A}_{s}
$$

where the transfer matrix $\mathbf{T}$ has dimension $2\left(2 N_{q}+1\right) \times 2\left(2 N_{q}+1\right)$, with $N_{q}$ being the truncation order employed for the summations in Eqs. (2) and (3). According to the impedance method, it is expressed as [46, 48]

$$
\mathbf{T}=\left[\mathbf{M}^{H K}\left(k_{p}, R_{2}\right)\right]^{-1}\left[\mathbf{Z}^{\text {scat }}+\mathbf{Z}^{\text {tot }}\right]^{-1}\left[\mathbf{Z}^{\text {inc }}-\mathbf{Z}^{\text {tot }}\right]\left[\mathbf{M}^{J I}\left(k_{p}, R_{2}\right)\right],
$$

where the additional matrix $\mathbf{M}$ contains some algebraic operations for Bessel functions, and the three impedance matrices $\mathbf{Z}^{\text {inc }}, \mathbf{Z}^{\text {scat }}$ and $\mathbf{Z}^{\text {tot }}$ are evaluated at the boundary $R=R_{2}$ for the incoming, scattered and total waves, respectively. In Eq. (8), $\mathbf{Z}^{\text {tot }}$ is the only one to be determined because it depends on the resonant structures. Following the procedures introduced in Refs. [46, 48], we obtain the remaining impedance matrix (see Appendix A)

$$
\mathbf{Z}_{q s}^{\text {tot }}=-\sum_{n=1}^{M} \frac{\mathrm{e}^{\mathrm{i}(s-q) \theta_{n}}}{2 \pi R_{2}} \mathbf{Z}
$$

where $\mathbf{Z}$ is an auxiliary impedance matrix introduced to simplify the expression.

Once the T-matrix is determined, the total displacement field in the background plate is easily available according to Eqs. (2) and (7). On this basis, the displacements in the inner plates and beams are determined by the following procedures:

$$
\left(\mathbf{C}_{n}\right)_{q}=\sum_{s=-\infty}^{\infty}\left(\mathbf{T}_{n}\right)_{q s}\left[\begin{array}{c}
W \\
W, R
\end{array}\right]_{s},
$$

where the sub-matrix $\left(\mathbf{T}_{n}\right)_{q s}$ is one element of the transfer matrix for internal waves localized in the $n$th plate $\mathbf{T}_{n}=\mathbf{L}_{n}^{-1} \mathbf{R}_{n}$. To be specific, $\mathbf{L}_{n}$ and $\mathbf{R}_{n}$ are block matrices with elements

$$
\begin{gathered}
\left(\mathbf{L}_{n}\right)_{q s}=2 \pi r_{1} \delta_{q s} \mathbf{N}_{s s}^{J I}\left(k_{i}, r_{1}\right)-\mathbf{K}_{11} \mathbf{M}_{s s}^{J I}\left(k_{i}, r_{1}\right) \mathrm{e}^{\mathrm{i}(s-q) \theta_{n}}, \\
\left(\mathbf{R}_{n}\right)_{q s}=\mathrm{e}^{\mathrm{i}(s-q) \theta_{n}} \mathbf{K}_{12},
\end{gathered}
$$


where $\delta$ and $\mathbf{K}$ are the Kronecker symbol and beam stiffness matrix, respectively. According to the boundary conditions in Eqs. (6a) and (6b), finally, the scattering problem is completely solved with

$$
\mathbf{D}_{n}=\sum_{q=-\infty}^{\infty} \mathbf{H}^{-1}\left[\begin{array}{c}
\mathbf{M}_{q q}^{J I}\left(k_{i}, r_{1}\right)\left(\mathbf{C}_{n}\right)_{q} \\
\mathbf{M}_{q q}^{J I}\left(k_{p}, R_{2}\right) \mathbf{A}_{q}+\mathbf{M}_{q q}^{H K}\left(k_{p}, R_{2}\right) \mathbf{B}_{q}
\end{array}\right] \mathrm{e}^{\mathrm{i} q \theta_{n}},
$$

where the column matrix $\mathbf{D}_{n}=\left[\begin{array}{llll}D_{1}^{n} & D_{2}^{n} & D_{3}^{n} & D_{4}^{n}\end{array}\right]^{t}$ contains the coefficients determining the flexural waves in the $n$th beam, and $\mathbf{H}$ is one auxiliary matrix employed in expressing the beam stiffness matrix. The reader is addressed to 165 Refs. [44, 46, 48] to get the explicit expressions of the auxiliary functions $\mathbf{H}$ as well as for any more details about the impedance method and the multiple scattering theory applied to flexural waves.

\subsection{Finite element simulations}

The accuracy of the semi-analytical method described above has been analyzed by studying the interaction of flexural waves with a cavity containing a single 1-beam resonator. A series of full wave simulations are performed within the framework of the finite element method. We have employed the solid mechanics module of the commercial package COMSOL Multiphysics, which solves the full elastic equations in three-dimensions (3D). Therefore, both in-plane and out-of-plane motions are provided by the finite element solutions.

We have considered three different resonant cavities drilled in an aluminum plate with thickness $h=1 \mathrm{~mm}$. For the numerical simulation, we have employed the following values as the material parameters of aluminum: Young's modulus $E=69 \mathrm{GPa}$, Poisson's ratio $\nu=0.33$, and density $\rho=2.7 \times 10^{3} \mathrm{~kg} \mathrm{~m}^{-3}$. As 180 the radius of the cavities [see Fig. 1(b)] we assume that $R_{2}=10 \mathrm{~mm}$. As the values of the geometrical parameters of the 1-beam resonators we consider that: $r_{1}=5 \mathrm{~mm}$ and $b=2 \mathrm{~mm}$. The beam length $\ell$ and the thickness of the circular plate $t$ vary with resonators. To be specific, we have studied the following three cases: (i) $\ell=8 \mathrm{~mm}, t=1 \mathrm{~mm}$; (ii) $\ell=8 \mathrm{~mm}, t=3 \mathrm{~mm}$; and

185 (iii) $\ell=1 \mathrm{~mm}, t=3 \mathrm{~mm}$. Each resonant cavity is placed at the center of an infinite aluminum plate and it is excited by a punctual source located at $(-2$, 2 ), where the coordinates are normalized to $R_{2}$.

Fig. 2 shows the snapshots of the out-of-plane displacements obtained from the $3 \mathrm{D}$ full wave simulations (left panels) and our 2D semi-analytical algorithm (right panels). The top, middle and bottom displacement patterns correspond to results of the three resonators with parameters specified in cases (i), (ii) and (iii), respectively. The resonant structures are excited by a punctual source emitting cylindrical waves with $k_{p} R_{2}=0.2 \pi$ in cases (i) and (ii), and $k_{p} R_{2}=0.26 \pi$ in case (iii). The comparison of the displacements patterns at the left and 195 right panels in Fig. 2 demonstrates that the scattering patterns obtained from the 3D finite-element solver are fairly good reproduced by our semi-analytical algorithm even in the extreme case illustrated in Figs. 2(e) and 2(f), where the resonator consists of an extremely short beam and a heavy mass. Therefore, we 
can conclude that our semi-analytic method is accurate enough to describe the

\subsection{Low frequency resonant bandgaps produced by lattices of $N$-beam resonators}

For its own interest and for comprehensiveness, this section is devoted to discuss the properties of the flexural band structures corresponding to square lattices of $N$-beam resonators. Fig. 3(a) depicts, as a typical example, the

The plots in Fig. 2 deserve more additional discussion. For example, the scattering patterns corresponding to resonators specified in cases (i) and (ii) exhibit appreciable differences, where the thickness of the inner plate is the only variable being changed. It is observed that for case (i), corresponding to the resonator with lighter mass, the displacement pattern displays features typical of a strong local resonance where the resonator vibrates much more heavily than the background plate. On the contrary, for case (ii), when the mass of the inner plate is heavier, Figs. 2(c) and 2(d) indicate that no resonant response seems to be excited. In this case, the resonant frequency shifts to a lower value (see Appendix B) and the inner structure vibrates with the background plate.

The plots in Figs. 2(e) and 2(f), corresponding to a resonator with an extremely short beam, show an interesting behavior not observed for longer beams. From the 3D full wave simulation shown in Fig. 2(e), we infer that the beam is twisted in accordance with the rotation motion observed in the inner plate. This effect, not reproduced in Fig. 2(f), is associated to the fact that the Euler-Bernoulli theory is unable to describe any type of torsional deformations. Therefore, our semi-analytical model might exhibit small discrepancies compared with full wave simulations for the case of 1-beam resonators with an extremely short beam.

\section{Results and discussion}

Our purpose here is showing that single-phase metamaterial plates based on lattices of resonant cavities containing a certain number $M$ of 1-beam resonators produce complete and broad resonant bandgaps at low frequencies. The flexural band structure of these metamaterials is obtained by using the $2 \mathrm{D}$ semianalytical model developed in Sec. 2.1 and the multiple scattering algorithm developed in Ref. [44]. To start with, we thoroughly investigate the band structure of cavity lattices containing $N$-beam resonators in order to show that 1-beam resonators are the right choice to get low frequency omnidirectional bandgaps. In a second step, it is shown that an increasing number $M$ of 1-beam resonators inside the cavity produce additional complete bandgaps. For the case of cavities containing four uniformly distributed 1-beam resonators, three complete bandgaps are opened at extremely low frequencies with two narrow pass-bands between them. As the final step, finite metamaterial slabs are studied to prove that they can be used as attenuation devices providing total suppression of flexural waves in certain broadband frequency ranges.

scheme of a square lattice of 2-beam resonators in real space together with the 
definitions of the high symmetry directions in the reciprocal space, where the shadowed region represents the irreducible Brillouin zone. In the lattice, all the resonators are structured with one beam pointing to the high symmetry direction OA. In the calculations, we have considered the following values of the parameters described in Fig. 1(a): $R_{1}=5 \mathrm{~mm}, R_{2}=10 \mathrm{~mm}$ and $b=2 \mathrm{~mm}$. Regarding the period of the square lattice, it is $d=25 \mathrm{~mm}$.

Fig. 4 shows the calculated band structures for cavity lattices containing $N$ beam resonators, with $N$ taking values from one (a) to four (d). The dispersion relations obtained from the 3D finite element solver (hollow circles) and the 2D multiple scattering algorithm (solid circles) are depicted for the sake of comparison. Let us point out that in the framework of the finite element method, full elastic equations are solved in 3D and, therefore, both in-plane and out-ofplane dispersion relations are obtained from the calculations. However, in Fig. 4 , bands corresponding to pure in-plane modes are not represented for simplicity.

It is observed that, in general, the bands obtained with our 2D model agree fairly well with those obtained with the finite element solver. Nevertheless, some discrepancies are observed at certain frequencies, as for the case of the bands labeled with points a to $\mathbf{d}$. Since the truncation order employed in the summations is large enough to guaranty adequate accuracy, the origin of these 260 discrepancies lies in the intrinsic limitation of our modeling. A calculation of the eigenmodes at points a to $\mathbf{d}$ indicates that at least one beam of the corresponding $\mathrm{N}$-beam resonator is twisted in order to coordinate with the rotation motion of the inner plates. As pointed out earlier, our model is based on the EulerBernoulli theory, which is unable to describe any torsional deformation and, 265 consequently, observable discrepancies are expected within bands containing modes of such type of deformations. For a comprehensive discussion of this effect, the reader is addressed to Ref. [44] where a 3D plot of mode labeled as $\mathbf{b}$ in Fig. 4(b) is given for the case of 2-beam resonators. Despite a few drawbacks, it must be stressed that our modeling is accurate enough to predict flexural270 related resonant bandgaps because they have no concern with the torsional deformation.

Let us discuss now the properties of the bands associated to pure out-of-plane (flexural) modes, which are obtained by our 2D algorithm and are described by solid circles in Fig. 4. Complete and partial bandgaps are opened because 275 of the interaction between the local resonances and the propagating waves in the host plate. Figure 4(a) provides a direct evidence that a narrow complete bandgap (normalized width $\approx 14.0 \%$ ) is opened because of the interaction of the flexural waves propagating in the plate with the fundamental mode of the 1-beam resonator, which is associated to the flat band passing through the point 280 e. To be specific, the bottom and upper edges of this complete low frequency resonant bandgap are 1.40 and $1.61 \mathrm{kHz}$, respectively. The fundamental mode of the 1-beam resonator is schematically described in Appendix B and its frequency can be obtained by using a simple model based on the spring-mass oscillator. It is observed from Fig. 4 that platonic crystals containing $N$-beam resonators 285 with $N \geq 2$ do not exhibit complete bandgaps. We must emphasize that this statement is not always to be true if the geometric symmetry is broken or 
the resonant frequency is tuned to much lower frequencies at the cost of using extremely heavy masses.

To obtain a physical insight of the reason explaining why lattices of 1-beam resonators produce complete bandgaps, the reader is addressed to Appendix C. In brief, a study of the interaction of a single $N$-beam resonator with impinging waves coming from different directions lets conclude that the structural anisotropy of the resonator suppresses its omnidirectional excitation for values $N \geq 2$. In addition, let us remark that the eigenfrequencies of the modes at the 295 points $\mathbf{e}$ to $\mathbf{h}$ in Fig. 4 are 1.40, 6.08, 8.71 and $10.19 \mathrm{kHz}$. These values are in good agreement with those extracted from Fig. C.1.

\subsection{Omnidirectional bandgaps produced by cavities containing multiple 1-beam resonators}

Inspired by the omnidirectional resonant bandgap created by the lattice of 1-beam resonators [see Fig. 4(a)], we foresee that additional 1-beam resonators into the cavity could produce a broadening of the bandgap or produce new complete gaps due to the increasing of degree-of-freedom (DOF) of the resonant system [26, 49-52]. However, restricted by the available space inside the circular cavity, a comprehensive analysis should be made regarding the dependence of the fundamental frequency with respect to the resonator parameters. Particularly, its dependence on the length of the beam $(\ell)$ and the radius $\left(r_{1}\right)$ and thickness of the circular plate $(t)$ has been comprehensively reported in Appendix D. The results of such dependence have been employed to choose the parameters providing the lowest resonant modes and keeping simultaneously

310 a good structural reliability. Here, it should be pointed out that the lowest resonant frequency hardly changes with the lattice period and the DOF of the resonant system, so the parametric study performed in Appendix D would make sense for the multi-DOF resonant system as well. Thus, the selected parameter values are: $R_{2}=10 \mathrm{~mm}, r_{1}=2.5 \mathrm{~mm}, e=4 \mathrm{~mm}$ and $b=2 \mathrm{~mm}$. The geomet315 rical meaning of these parameters is described in Fig. 1(b) for the case of two 1-beam resonators $(M=2)$. In addition, we have considered that the thickness of the smaller plates is $t=3 \mathrm{~mm}$ and that the resonant cavities are arranged in a square lattice with period $d=25 \mathrm{~mm}$.

Fig. 5 depicts the band structures obtained for lattices of cavities containing 320 multiple 1-beam resonators with $M$ taking values from one (a) to four (d). Note that $M=4$ is the maximum number of 1-beam resonators that can be embedded in the cavity with the geometrical parameters chosen above. The irreducible Brillouin zone and the high symmetry directions in reciprocal space are shown in Fig. 3(b) for cavities containing two 1-beam resonators, as an example. All 325 the bands are calculated within the framework of the multiple scattering method [44]. They are obtained at frequencies below $10 \mathrm{kHz}$, where the accuracy of the method has been demonstrated. For comparison purposes, the hollow circles in Fig. 5(a) describe the band structure of flexural waves propagating in a platonic crystal made of free holes without embedded resonators. The dispersion 
On the one hand, Fig. 5(a) shows that a square lattice of cavities with a single 1-beam resonator produces a dispersion relation (solid circles) strongly different to the case in which the cavities have no resonators (hollow circles). The differences can be easily explained in terms of the cavity-embedded resonance, which strongly interacts with the flexural waves propagating in the host plate. The cavity resonance produces a completely new band in the band structure (labeled as 1) and generates an omnidirectional bandgap. The frequencies determining this complete bandgap, with normalized width $\approx 16.5 \%$, are 2.61 $\mathrm{kHz}$ and $3.08 \mathrm{kHz}$ for the lower and upper edges, respectively.

The behavior explained above is basically reproduced for the cavities containing a higher number of 1-beam resonators. For $M=2$, Fig. 5(b) shows that two additional bands (labeled as 1 and 2) appear in comparison with the case of non-resonant cavities. Three and four additional bands appear for the cases $M=3$ and $M=4$ in Figs. 5(c) and 5(d), respectively. On the other hand, it is also noticeable that the band structures exhibit additional omnidirectional bandgaps because of the increasing number of 1-beam resonators inside the cavity. Two omnidirectional bandgaps are observed for the case $M=3$ and three appear for the case $M=4$. However, the case $M=2$ is singular since only one omnidirectional bandgap is observed and it is narrower than that obtained for 350 the case $M=1$.

The band structure in Fig. 5(d), corresponding to $M=4$, is more relevant because of its potential application in designing structures for broadband attenuation of flexural waves in plates. Notice that three complete bandgaps are shown: the 1st covers the frequency range from $2.76 \mathrm{kHz}$ to $3.01 \mathrm{kHz}$ (normalized 355 width $\approx 8.7 \%$ ), the 2 nd goes from $3.21 \mathrm{kHz}$ to $4.55 \mathrm{kHz}$ (normalized width $\approx$ $34.5 \%$ ), and the third extends from $5.30 \mathrm{kHz}$ till $6.87 \mathrm{kHz}$ (normalized width $\approx$ $25.8 \%$ ). Since the bandwidths of the 2 nd gap and the 3rd gap are, respectively, one-half of octave band and one-third of octave band, they can be considered as broad bandgaps according to engineering acoustics. More recently, it has been 360 demonstrated that the resonant bandgaps induced by multi-DOF resonators can be combined into a broadband wave attenuation region by employing intrinsic damping of the constitutive materials [52]. This strategy cannot be introduced directly in this design because of the negligible damping of metal plates. However, as shall be shown later (see Fig. 11), it is expected that the transmitted waves in the narrow passbands, which are associated to the local resonances, would be strongly suppressed by using composite platonic crystal slab, consisting of several slabs with complementary bandgaps by slightly changing the geometrical parameters.

Now, let us discuss in brief the resonant features of the cavities containing multiple 1-beam resonators. Fig. 6 displays the eigenmodes for two and four 1-beam resonators at selected points of the band structure; i.e., at the points a to $\mathbf{f}$ in Fig. 5. Obviously, all of them are closely related with the fundamental resonance of the 1-beam resonator; they can be considered as combinations of the fundamental resonance. Thus, for the case $M=2$, Fig. 6(a) depicts the 375 low frequency mode, with frequency $2.71 \mathrm{kHz}$. It is labeled as a in Fig. 5(a), and the eigenmode presents two 1-beam resonators oscillating out of phase. 
On the contrary, the two resonators oscillate in phase for the mode shown in Fig. 6(b), which has frequency $3.91 \mathrm{kHz}$ and defines the high frequency mode. Unfortunately, the interaction of these resonances with the flexural waves in the plate produces an extremely narrow bandgap (normalized width $\approx 5.3 \%$ ), extending from 2.76 to $2.91 \mathrm{kHz}$. However, when another two resonators are added in each cavity, the band structure in Fig. 5(d) shows three almost adjacent complete bandgaps.

The eigenmodes at points $\mathbf{c}$ to $\mathbf{f}$ in the band structure are depicted in Figs. ${ }_{385} 6(\mathrm{c})$ to $6(\mathrm{f})$. It is observed that the modes differ in the phase of the oscillations of the individual 1-beam resonators. These modes are helpful to create complete gaps with broad bandwidth, as it is shown in the band structure. As a summary of the discussion above, we can say that resonant cavities containing four 1-beam resonators $(M=4)$ are the proper choice for implementing devices to attenuate flexural waves in a broad frequency range.

Based on the previous analysis, a parametric study has been performed for the case $M=4$ to investigate the influence of the geometrical parameters on the resonant bandgaps. The results are shown in Fig. 7 where the left, middle and right panels provide the evolution process (upper panels) and the normalized 395 width (lower panels) of the complete bandgaps changing with the lattice period $d$, the beam length $\ell$ and the thickness of the smaller plates $t$, respectively. Only one parameter is set as variable in each plot, and the other parameter values are the same as those employed in the calculation of Fig. 5.

From Figs. 7(a) and 7(d), we observe that the 2nd and 3rd bandgaps get narrower and narrower with the increasing of lattice period. During this process, only slightly variation is observed for the lower edges of the 1st and 2nd bandgaps, nevertheless, it decreases a lot for the 3rd bandgap. This indicates that, except the geometrical parameters of the resonators, the spacing between them will also influence the splitting of resonant frequencies for multiple 1-beam 405 resonators. With the increasing of the lattice period, the interaction between the local resonators coming from different unit cells gets weaker, and then the resonant frequencies tend to be the values of a multiple 1-beam resonator embedded in an infinite plate, approaching the resonant frequency of a single 1-beam resonator. We conclude from the analysis that smaller unit cell dimension is preferred in order to get wider bandgaps.

In the last two cases [see Figs. 7(b) and 7(c)], corresponding to resonators varied in the beam length $\ell$ and mass thickness $t$, it is seen that both the positions and bandwidths of the gaps are influenced by the geometrical size. In the variation range, the width of 1st bandgap is almost unchanged (normalized width $\approx 8 \%$ to $10 \%$ ), nevertheless, by selecting proper geometrical parameters we are able to enlarge the 2nd and 3rd bandgaps. For example, the normalized width reaches $36.4 \%$ and $35.9 \%$, respectively, for the 2nd and 3rd bandgaps when $\ell / R_{2}$ taking value 0.275 . 


\subsection{Broadband attenuation by metamaterial slabs made of cavities with multiple} 1-beam resonators

To support the previous results relative to the complete bandgap formation by lattices of resonant cavities, we have performed numerical experiments analyzing the vibration transmission through different finite metamaterial slabs. The simulations are performed in the framework of the multiple scattering 425 method described in Ref. [44] together with the T-matrix introduced in Sec. 2.1. We have studied metamaterial slabs with a fix number of 12 rows in the direction perpendicular to the impinging waves. The parameter values used here are the same as those employed in the calculation of Fig. 5. Moreover, the thickness of the slab along the propagation direction has been increased until obtaining total suppression of the transmitted waves at the frequency regions where omnidirectional bandgaps are predicted. For the case of metamaterials based on cavities containing 1-beam resonators, a complete suppression has been obtained on the slab back side when its thickness reaches 8 layers. However, for the case of four 1-beam resonators in each cavity, only 5 layers are needed.

As an example, Fig. 8 shows snapshots of the out-of-plane displacements calculated by considering cylindrical waves interacting with a metamaterial slab made of $5 \times 12$ resonant cavities. Each cavity contains four 1-beam resonators. The cylindrical waves are generated by a punctual source located at $(-2,0)$ in units normalized to the lattice period, $d$. The displacement patterns shown in

${ }_{440}$ Fig. 8(a) correspond to impinging waves with frequency $1.8 \mathrm{kHz}$ [i.e., within a pseudogap in Fig. 5(d)], while those in Fig. 8(b) correspond to a frequency of $3.6 \mathrm{kHz}$, within a complete bandgap. For the last frequency, it is observed that incident waves are fully reflected by the slab. However, for the frequency in the pseudogap, waves are partially transmitted, producing a complex displacement pattern on the slab back side.

From the displacement patterns shown in Fig. 8, it is observed that the waves diffracted by the borders of the slab might interfere with the waves transmitted through the slab, resulting in a reduction of the attenuation by the slab. To evaluate this effect we have considered the amplitude of the excited and ${ }_{450}$ response signals, which are expressed as $W_{e}$ and $W_{r}$, respectively. Therefore, the total vibration transmission at a given point can be obtained by calculating the ratio $T=\left|W_{r} / W_{e}\right|$, which is a frequency dependent function. This ratio may reach values greater than unity since the response signal contains the possible interference with diffracted waves. It has been calculated at three different observation points behind the slab, as it is schematically explained in Fig. 9.

Fig. 10 plots the frequency dependence of the vibration transmission $T(\omega)$ for two types of resonant cavities. The transmission spectra are calculated at the three selected points; i.e., at the positions labeled as 1, 2 and 3 in Fig. 9. Fig. 10(a) represents results obtained for the case of resonant cavities contain460 ing just a single 1-beam resonator. It is observed that, for certain frequencies, the transmission takes values higher than unity. This effect is mostly due to interference effect, in which the signal crossing directly through the slab interferes constructively at the observational point with the waves diffracted by its borders. 
For the case of resonant cavities containing four 1-beam resonators, the transmission spectra in Fig. 10(b) clearly show three valleys with zero transmission. As in the previous case, the bandwidths of the valleys are in good agreement with the three complete bandgaps predicted in Fig. 5(d). The bandgaps seem to be completely formed since their transmission bandwidths

470 don't depend on the observational point. However, the profile of the valley corresponding to the bandgap with largest frequencies shows a slight dependence with the observational points and, therefore, an interference with the diffracted waves seem to play a non negligible effect. For frequencies around the valleys with zero transmission, the interference effect seems to be particularly strong,

475 giving transmission values larger than unity. This constructive effect is remarkable at position 1 , where the diffracted waves arrive in phase.

The results shown in Fig. 10 prove the existence of complete bandgaps. However, for the case of cavities containing four 1-beam resonators, a more practical question is that can we combine these separated bandgaps into a broadband wave attenuation region. More recently, Barnhart and co-workers [52] provided a solution by using dissipative multi-resonators. This mechanism fails in our scheme because the damping of aluminum plate is negligible to make difference. By combining two or more metamaterial slabs together, however, the goal might be achieved if their multi-bandgaps are complementary to each other. To prove

485 this, we consider a composite slab composed of three metamaterial slabs made of resonant cavities differing in their beam length $\ell$. To be specific, the composite slab consists of a fix number of 12 rows in the $y$-direction, and it totally contains 9 layers in the $x$-direction. From left to right, the resonators in each three layers have the same geometrical parameters and the beam length, $\ell / R_{2}$, takes values $0.35,0.3$ and 0.25 , respectively. Fig. 11 plots the frequency dependence of the vibration transmission for flexural waves through the composite slab. As expected, the multiple bandgaps effectively merge into a broadband valley with zero transmission. More specific, the transmission valley extends from $2.80 \mathrm{kHz}$ to $7.91 \mathrm{kHz}$ with normalized width $\approx 95.3 \%$, representing an enhancement of 495 about one order of magnitude compared with the absolute bandwidth obtained for the case of a single 1-beam resonator in the cavity. These results are encouraging and support our claim of proposing this type of structures for broadband vibration suppression at low frequencies.

\section{Conclusions}

In summary, we have studied the response of metamaterial plates made of two types of resonant cavities. First, the response of a square lattice of cavities consisting of $N$-beam resonators, with $1 \leq N \leq 4$, leads to conclude that 1-beam resonators are capable of creating omnidirectional bandgaps. A physical insight of this result was provided by analyzing the scattering cross-section maps of a 505 single resonant cavity, reveling that the cavity containing 1-beam resonator is the easier one giving an omnidirectional resonant response at low frequencies. This property gives the 1-beam resonators significant advantages in constructing metamaterial plates for omnidirectional wave shielding. Consequently, in order 
to widen complete bandgaps, we have also studied lattices of resonant cavities containing a multiple number $M$ of 1-beam resonators. This new type of cavity lattices were theoretically studied by employing the impedance method, with a T-matrix here implemented, and the multiple scattering theory [44]. Simulations show that an increasing number of 1-beam resonators in the cavity usually results in an increasing number of complete bandgaps with broad bandwidth.

515 Particularly, for $M=4$, it is demonstrated that the absolute bandwidth is broadened by about one order of magnitude compared with the bandgap obtained for the case of a single 1-beam resonator. As a potential application of this extraordinary effect, we have computed the vibration transmission for finite metamaterial slabs, showing that the transmission valleys match fairly well with ${ }_{520}$ the complete resonant bandgaps predicted in the band structure. The resonant bandgaps can be easily tuned to lower frequencies by changing the geometrical parameters, such as the length of the beams, the radius and thickness of the inner plates. This work will do a benefit for the fabrication of single-phase structures providing vibration shielding in plates with low cost and high reliability.

${ }_{525}$ To conclude, we believe that this type of simple and light-weight structured plates may have potential applications in aeronautic and astronautic industries for broadband vibration suppression at low frequencies.

\section{Acknowledgments}

This work was supported by the Ministerio de Economía y Competitividad of 530 the Spanish government and the European Union Fondo Europeo de Desarrollo Regional (FEDER) [Grant No. TEC2014-53088-C3-1-R], and the National Natural Science Foundation of China [Grant Nos. 11432004 and 11421091]. Penglin Gao acknowledges a scholarship provided by China Scholarship Council [Grant No. 201606120070].

\section{Appendix A. Impedance matrix for total waves: $\mathrm{Z}^{\text {tot }}$}

Following the procedures developed in Refs. [46, 48], the remaining impedance matrix $\mathbf{Z}^{\text {tot }}$ is derived in this Appendix. For simplicity, the key steps are provided only, and the reader is addressed to the original papers for a complete derivation.

The radial moment and Kirchhoff stress can be expressed in multipole expansion form after introducing the series defined in Eqs. (2) and (3). At the boundary of the $n$th plate $r=r_{1}$, we obtain the following relationship:

$$
\left[\begin{array}{c}
M_{r}^{n} \\
V_{r}^{n}
\end{array}\right]_{q}=\left(\mathbf{Y}_{q q}\right)^{-1}\left[\begin{array}{c}
W^{n} \\
W_{, r}^{n}
\end{array}\right]_{q}
$$

with notations $\mathbf{Y}_{q q}=\mathbf{M}_{q q}^{J I}\left(k_{i}, r_{1}\right)\left[\mathbf{N}_{q q}^{J I}\left(k_{i}, r_{1}\right)\right]^{-1}$ and $W_{, r}^{n}=\partial W^{n} / \partial r$. The matrix $\mathbf{N}$ involves complex operations for the Bessel functions. Its expression 
can be found in Ref. [46]. Combining Eq. (A.1) with the boundary conditions in Eqs. (6c) and (6d) (left column), we have

$$
\left[\begin{array}{c}
W^{n} \\
W_{, r}^{n}
\end{array}\right]_{q}=\frac{\mathrm{e}^{-\mathrm{i} q \theta_{n}}}{2 \pi r_{1}} \mathbf{Y}_{q q}\left[\begin{array}{c}
M^{n}\left(r_{1}\right) \\
Q^{n}\left(r_{1}\right)
\end{array}\right]
$$

From the boundary conditions in Eqs. (6a) and (6b) (left column), the displacement and slope of the $n$th beam can be expressed as function of bending moment and shear force

$$
\left[\begin{array}{c}
V^{n}\left(r_{1}\right) \\
V_{, x_{n}}^{n}\left(r_{1}\right)
\end{array}\right]=\mathbf{Y}\left[\begin{array}{l}
M^{n}\left(r_{1}\right) \\
Q^{n}\left(r_{1}\right)
\end{array}\right]
$$

with notation

$$
\mathbf{Y}=\frac{1}{2 \pi r_{1}} \sum_{q=-\infty}^{\infty} \mathbf{Y}_{q q}
$$

Substituting the stiffness matrix K [46] into Eq. (A.3), after grouping we obtain the following equation:

$$
\left[\begin{array}{l}
M^{n}\left(r_{2}\right) \\
Q^{n}\left(r_{2}\right)
\end{array}\right]=\mathbf{Z}\left[\begin{array}{c}
V^{n}\left(r_{2}\right) \\
V_{, x_{n}}^{n}\left(r_{2}\right)
\end{array}\right]
$$

where the auxiliary impedance matrix is

$$
\mathbf{Z}=\left[\mathbf{K}_{21}^{-1}-\mathbf{Y} \mathbf{K}_{11} \mathbf{K}_{21}^{-1}\right]^{-1}\left[\mathbf{K}_{21}^{-1} \mathbf{K}_{22}+\mathbf{Y}\left(\mathbf{K}_{12}-\mathbf{K}_{11} \mathbf{K}_{21}^{-1} \mathbf{K}_{22}\right)\right] .
$$

Expanding the boundary conditions in Eqs. (6c) and (6d) (right column) in azimuthal order and casting them into Eq. (A.5), we obtain

$$
\left[\begin{array}{c}
M_{R} \\
V_{R}
\end{array}\right]_{q}^{\mathrm{tot}}=\sum_{n=1}^{M} \frac{\mathrm{e}^{-\mathrm{i} q \theta_{n}}}{2 \pi R_{2}} \mathbf{Z}\left[\begin{array}{c}
V^{n}\left(r_{2}\right) \\
V_{, x_{n}}^{n}\left(r_{2}\right)
\end{array}\right] .
$$

Combining Eq. (A.7) with the remaining boundary conditions in Eqs. (6a) and (6b) (right column), we have

$$
\left[\begin{array}{c}
M_{R} \\
V_{R}
\end{array}\right]_{q}^{\mathrm{tot}}=\sum_{s=-\infty}^{\infty} \sum_{n=1}^{M} \frac{\mathrm{e}^{\mathrm{i}(s-q) \theta_{n}}}{2 \pi R_{2}} \mathbf{Z}\left[\begin{array}{c}
W \\
W_{, R}
\end{array}\right]_{s}^{\mathrm{tot}} .
$$

Finally, the $2 \times 2$ blocks of the impedance matrix for the total waves are expressed as

$$
\mathbf{Z}_{q s}^{\mathrm{tot}}=-\sum_{n=1}^{M} \frac{\mathrm{e}^{\mathrm{i}(s-q) \theta_{n}}}{2 \pi R_{2}} \mathbf{Z}
$$


It is demonstrated in Sec. 3 that the fundamental resonance plays an important role in opening low frequency bandgaps. When the flexural resonance occurs, the 1-beam resonator behaves like a simple spring-mass oscillator. Its resonant frequency can be predicted by the well-known formula [44]

$$
f=\frac{1}{2 \pi} \sqrt{\frac{K_{e}}{M}}
$$

as illustrated in Fig. B.1(a), where $M$ and $K_{e}$ represent the mass of the inner plate and the effective stiffness of the beam, respectively.

Once the resonator is excited, the beam bends up and down under the action of the inner plate. In our modeling, the bending effect caused by the distributed inertia force is equivalent to a concentrated force applied at an equivalent point located on the segment AB [see Fig. B.1(b)]. A Cartesian coordinates $O-x y z$ is built at the anchor point connected to the background plate. The out-of-plane displacement of the beam is governed by the following equation of motion:

$$
E I \frac{\mathrm{d}^{2} w}{\mathrm{~d} x^{2}}=F\left(x_{0}-x\right), \quad x \in[0, \ell]
$$

where $x_{0}$ defines the location of the equivalent point within the range $r_{2}<x_{0}<$ $r_{1}+r_{2}$. For longer beams, the anchor point connected to the background plate is reasonable to be considered as clamped because the resonator vibrates much more heavily than the background plate when the resonance occurs. On this assumption, we have the following boundary conditions:

$$
\left.w\right|_{x=0}=0 \quad \text { and }\left.\quad \frac{\mathrm{d} w}{\mathrm{~d} x}\right|_{x=0}=0 .
$$

Combining Eqs. (B.2) and (B.3), we obtain the displacement of the equivalent point

$$
w_{0}=\frac{F}{E I}\left[\frac{1}{2} x_{0} \ell^{2}-\frac{1}{6} \ell^{3}+\left(x_{0}-\ell\right)\left(x_{0} \ell-\frac{1}{2} \ell^{2}\right)\right] .
$$

Finally, the resonant frequency is available by substituting the following effective parameters into Eq. (B.1):

$$
K_{e}=\frac{F}{w_{0}} \quad \text { and } \quad M=\pi \rho t r_{1}^{2} .
$$

Notice that the position of the equivalent point is not well defined, therefore no specific value can be obtained from Eq. (B.1). Nevertheless, it bounds the upper and lower limits of the resonant frequency when the minimum and maximum displacements are taken into consideration, respectively. In numerical calculations, we prefer to use the averaged displacements to get the approximate solutions. 


\section{Appendix C. Resonant response of a cavity with a $N$-beam resonator}

Consider a $N$-beam resonator located in an infinite plate like in Fig. 1(a). Let us consider that the resonant structure interacts with a plane wave propagating along the horizontal axis, which defines the positive $x$-direction. The scattering cross-section $\sigma_{\mathrm{sc}}$, which is defined as the ratio of the energy flux scattered to the incoming one, is employed here to characterize the resonant response of a cavity containing a $N$-beam resonator. The expression is [46]

$$
\sigma_{\mathrm{sc}}\left(k_{p} R_{2} / \pi, \theta_{n}\right)=-\frac{4}{k_{p}} \sum_{q=-\infty}^{\infty} \operatorname{Re}\left[(-\mathrm{i})^{q} B_{q}^{H}\right],
$$

550

where $B_{q}^{H}$ is the expansion coefficient for the scattered propagating waves.

The color maps depicted in Fig. C.1 represent the scattering cross-section obtained for a single cavity containing a $N$-beam resonator, with $N$ going from one to four. The horizontal and vertical axes represent the two variables involved in Eq. (C.1), i.e., the reduced frequency $k_{p} R_{2} / \pi$ and the inclination angle of one particular beam $\theta_{n}$, respectively. In each plot, the tilt angle of the first beam $\theta_{1}$ is annotated in the inset for illustration. It is observed that a series of narrow ridges come into being with the increasing of the excitation frequency, indicating the occurrence of some kinds of local resonances. This feature is apparently seen in Fig. C.1 where most of the ridges do not cover the whole angular range. Consider the case of a 2-beam resonator as an example. In this case, Fig. C.1(b) indicates that the fundamental resonance is excited when both beams are aligned perpendicularly to the incident direction (i.e., $\theta_{1}=90^{\circ}$ ), corresponding to label $\mathbf{b}$. However, the result is just the opposite in the parallel case $\left(\theta_{1}=0^{\circ}\right)$. If one wants to excite the higher-order resonance, however, it is better to place the 2-beam resonator with an inclination angle $\theta_{1}=45^{\circ}$, as described by label $\mathbf{f}$ in the figure.

From Fig. C.1 we observe that the number of beams has a significant influence on the frequency of the fundamental resonance. In reduced units, the fundamental frequencies of the $N$-beam resonators are 0.247 (a), 0.504 (b), 0.599 (c) and $0.648(\mathrm{~d})$, respectively. The corresponding values in absolute units are $1.48,6.18,8.71$ and $10.20 \mathrm{kHz}$ for the points a to $\mathbf{d}$, respectively. The observed blue shift is expected by analogy to a simple spring-mass oscillator whose effective stiffness increases with the number of beams. In addition, the number of beams influences the intensity of the resonant response as well. Based on the 575 previous results, we conclude that the 1-beam resonator provides an omnidirectional resonant response at low frequencies. As a consequence, metamaterials based on lattices of 1-beam resonators will produce complete resonant bandgaps in the dispersion relation.

\section{Appendix D. Tunability of the fundamental resonance of the 1-beam resonators}

This appendix is devoted to show that the fundamental frequency of the 1-beam resonator can be tuned into low-frequency regions by changing the ge- 
ometrical parameters, such as the beam length $(\ell)$, the thickness $(t)$ and radius $\left(r_{1}\right)$ of the inner plate. These parameters are used as variables in the calculation. For the rest of parameters, their values are equal to those employed in Sec. 2.2. It should be stressed that, for multiple 1-beam resonators, the resonant frequencies also depend on the spacing and DOF of the resonators [see Figs. 5 and 7(a)]. Nevertheless, the lowest one, approaching the resonant frequency of a single 1-beam resonator, can hardly be changed and thus the following parametric 590 study would make sense for the multi-DOF resonant system as well.

Fig. D.1 shows maps describing the dependence of the fundamental resonant frequency (in reduced units) on $\ell$ and $r_{1}$ for three thickness values: (a) $t=h$, (b) $t=3 h$, and (c) $t=5 h$. The results are extracted from the maps of scattering cross-section, similar to those depicted in Fig. C.1, for a single 1-beam resonator with given values for $\ell$ and $r_{1}$. Since $\ell$ and $r_{1}$ are subjected to the size of the circular hole, only the lower-left panels are occupied with results. The design space shrinks dramatically if more resonators are introduced into the hole, see the boundaries annotated with the number of 1-beam resonators 2, 3 and 4. It is concluded from Fig. D.1 that there is a negative correlation 600 between the resonant frequency and the parameters studied. As demonstrated in Appendix B, the 1-beam resonator shows a feature of spring-mass like oscillation in which the beam and inner plate serve as the spring and mass, respectively. Therefore, the increasing of $\ell$ reduces the effective stiffness $K_{e}$; the increasing of $r_{1}$ and $t$ adds the mass $M$ of the inner plate and, finally, the resonant frequency 605 lowers. The lowest frequency achieved in Fig. D.1 is about 0.1 in reduced units, corresponding to $\lambda=20 R_{2}$ and reaching the deep-subwavelength scale.

By modeling the 1-beam resonator as a spring-mass oscillator, a simple formula can be derived to predict the resonant frequency (see Appendix B). The formula given by Eq. (B.1) is here verified by changing the beam length and the thickness of the inner plate independently. The radius $r_{1}$ has little interest because of its poor tunability in controlling the frequency. Its value has been fixed to $r_{1}=4 \mathrm{~mm}$.

Fig. D.2 shows the dependence of the frequency (in reduced units) on $\ell$ (a) and $t(\mathrm{~b})$. The fixed parameters employed in the calculations are $t=1 \mathrm{~mm}$ ${ }_{615}$ in Fig. D.2(a) and $\ell=10 \mathrm{~mm}$ in Fig. D.2(b). The solid lines represent the frequencies obtained from the semi-analytical algorithm while the other lines are obtained with the simple spring-mass model. As explained in Appendix B, the position of the equivalent point is not well defined, and its extreme positions bound the limits of the resonant frequency. The dashed lines correspond to the approximate solutions when the averaged displacement is employed in the calculation. It is observed from Fig. D.2(a) that, if $\ell$ is greater than $0.4 R_{2}$, the frequency predicted by the spring-mass model agrees fairly well with the semi-analytical model described in Sec. 2. This statement is also supported by results in Fig. D.2(b) where the dashed and solid lines coincide for any value ${ }_{625}$ of $t$. For shorter beams, however, appreciable discrepancies appear because the end of the beam cannot be simply treated as clamped. The reader is addressed to Figs. 2(a) and 2(e) for the comparison where the fundamental resonance is excited for both cases. 


\section{References}

[1] A. Kheliff, A. E. Adibi, Phononic Crystals: Fundamentals and Applications, Springer, New York, 2016.

[2] T.-T. Wu, J.-C. Hsu, J.-H. Sun, Phononic plate waves, IEEE Trans. Ultrason., Ferroelectr., Freq. Control 58 (10) (2011) 2146-2161.

[3] P. A. Deymier, Acoustic Metamaterials and Phononic Crystals, Springer, London, 2013.

[4] X. Zhou, G. Hu, Analytic model of elastic metamaterials with local resonances, Phys. Rev. B 79 (19) (2009) 195109.

[5] D. Torrent, Y. Pennec, B. Djafari-Rouhani, Effective medium theory for elastic metamaterials in thin elastic plates, Phys. Rev. B 90 (10) (2014) 104110 .

[6] J. H. Oh, H. M. Seung, Y. Y. Kim, Doubly negative isotropic elastic metamaterial for sub-wavelength focusing: design and realization, J. Sound Vib. 410 (2017) 169-186.

[7] H. Huang, C. Sun, G. Huang, On the negative effective mass density in acoustic metamaterials, Int. J. Eng. Sci. 47 (4) (2009) 610-617.

[8] R. Graciá-Salgado, V. M. García-Chocano, D. Torrent, J. Sánchez-Dehesa, Negative mass density and $\rho$-near-zero quasi-two-dimensional metamaterials: design and applications, Phys. Rev. B 88 (2) (2013) 224305.

[9] Z. Liu, X. Zhang, Y. Mao, Y. Zhu, Z. Yang, C. T. Chan, P. Sheng, Locally resonant sonic materials, Science 289 (5485) (2000) 1734-1736.

[10] R. Zhu, X. Liu, G. Hu, C. Sun, G. Huang, Negative refraction of elastic waves at the deep-subwavelength scale in a single-phase metamaterial, Nat. Commun. 5 (2014) 5510.

[11] J. H. Oh, H. Min Seung, Y. Young Kim, A truly hyperbolic elastic metamaterial lens, Appl. Phys. Lett. 104 (7) (2014) 073503.

[12] E. Andreassen, K. Manktelow, M. Ruzzene, Directional bending wave propagation in periodically perforated plates, J. Sound Vib. 335 (2015) 187-203.

[13] R. Zhu, X. Liu, G. Hu, F. Yuan, G. Huang, Microstructural designs of plate-type elastic metamaterial and their potential applications: a review, Int. J. Smart Nano Mater. 6 (1) (2015) 14-40.

[14] X. Liu, G. Hu, Elastic metamaterials making use of chirality: a review, Strojniški vestnik-J. Mech. Eng. 62 (7-8) (2016) 403-418.

[15] Z. Yang, H. Dai, N. Chan, G. Ma, P. Sheng, Acoustic metamaterial panels for sound attenuation in the 50-1000 Hz regime, Appl. Phys. Lett. 96 (4) (2010) 041906. 
[16] P. Wang, F. Casadei, S. Shan, J. C. Weaver, K. Bertoldi, Harnessing buckling to design tunable locally resonant acoustic metamaterials, Phys. Rev. Lett. 113 (1) (2014) 014301.

[17] C. Claeys, E. Deckers, B. Pluymers, W. Desmet, A lightweight vibroacoustic metamaterial demonstrator: numerical and experimental investigation, Mech. Syst. Signal Process. 70 (2016) 853-880.

[18] Y. Wu, K. Yu, L. Yang, R. Zhao, X. Shi, K. Tian, Effect of thermal stresses on frequency band structures of elastic metamaterial plates, J. Sound Vib. 413 (2018) 101-119.

[19] U. Aridogan, I. Basdogan, A review of active vibration and noise suppression of plate-like structures with piezoelectric transducers, J. Intell. Mater. Syst. Struct. 26 (12) (2015) 1455-1476.

[20] R. McPhedran, A. Movchan, N. Movchan, Platonic crystals: bloch bands, neutrality and defects, Mech. Mat. 41 (4) (2009) 356-363.

[21] D.-L. Yu, Y.-Z. Liu, J. Qiu, H.-G. Zhao, Z.-M. Liu, Experimental and theoretical research on the vibrational gaps in two-dimensional three-component composite thin plates, Chin. Phys. Lett. 22 (8) (2005) 1958.

[22] J.-C. Hsu, T.-T. Wu, Lamb waves in binary locally resonant phononic plates with two-dimensional lattices, Appl. Phys. Lett. 90 (20) (2007) 201904.

[23] Y. Xiao, J. Wen, X. Wen, Flexural wave band gaps in locally resonant thin plates with periodically attached spring-mass resonators, J. Phys. D: Appl. Phys. 45 (19) (2012) 195401.

[24] Y. Xiao, J. Wen, X. Wen, Sound transmission loss of metamaterial-based thin plates with multiple subwavelength arrays of attached resonators, J. Sound Vib. 331 (25) (2012) 5408-5423.

[25] M. Nouh, O. Aldraihem, A. Baz, Wave propagation in metamaterial plates with periodic local resonances, J. Sound Vib. 341 (2015) 53-73.

[26] H. Peng, P. F. Pai, H. Deng, Acoustic multi-stopband metamaterial plates design for broadband elastic wave absorption and vibration suppression, Int. J. Mech. Sci. 103 (2015) 104-114.

[27] A. Nateghi, L. Van Belle, C. Claeys, E. Deckers, B. Pluymers, W. Desmet, Wave propagation in locally resonant cylindrically curved metamaterial panels, Int. J. Mech. Sci. 127 (2016) 73-90.

[28] T.-T. Wu, Z.-G. Huang, T.-C. Tsai, T.-C. Wu, Evidence of complete band gap and resonances in a plate with periodic stubbed surface, Appl. Phys. Lett. 93 (11) (2008) 111902. 
[29] Y. Pennec, B. Djafari-Rouhani, H. Larabi, J. Vasseur, A. Hladky-Hennion, Low-frequency gaps in a phononic crystal constituted of cylindrical dots deposited on a thin homogeneous plate, Phys. Rev. B 78 (10) (2008) 104105.

[30] M. Oudich, Y. Li, B. M. Assouar, Z. Hou, A sonic band gap based on the locally resonant phononic plates with stubs, New J. Phys. 12 (8) (2010) 083049 .

[31] M. Oudich, M. Senesi, M. B. Assouar, M. Ruzenne, J.-H. Sun, B. Vincent, Z. Hou, T.-T. Wu, Experimental evidence of locally resonant sonic band gap in two-dimensional phononic stubbed plates, Phys. Rev. B 84 (16) (2011) 165136.

[32] Y. Xiao, J. Wen, L. Huang, X. Wen, Analysis and experimental realization of locally resonant phononic plates carrying a periodic array of beam-like resonators, J. Phys. D: Appl. Phys. 47 (4) (2013) 045307.

[33] M. Badreddine Assouar, M. Oudich, Enlargement of a locally resonant sonic band gap by using double-sides stubbed phononic plates, Appl. Phys. Lett. 100 (12) (2012) 123506.

[34] Y. Li, T. Chen, X. Wang, Y. Xi, Q. Liang, Enlargement of locally resonant sonic band gap by using composite plate-type acoustic metamaterial, Phys. Lett. A 379 (5) (2015) 412-416.

[35] X. Fang, J. Wen, B. Bonello, J. Yin, D. Yu, Ultra-low and ultra-broad-band nonlinear acoustic metamaterials, Nat. Commun. 8 (1) (2017) 1288.

[36] J. H. Oh, S. Qi, Y. Y. Kim, B. Assouar, Elastic metamaterial insulator for broadband low-frequency flexural vibration shielding, Phys. Rev. Appl. 8 (5) (2017) 054034.

[37] Y.-F. Wang, Y.-S. Wang, Complete bandgap in three-dimensional holey phononic crystals with resonators, J. Vib. Acoust. 135 (4) (2013) 041009.

[38] Y.-F. Wang, Y.-S. Wang, Multiple wide complete bandgaps of twodimensional phononic crystal slabs with cross-like holes, J. Sound Vib. 332 (8) (2013) 2019-2037.

[39] M. Farhat, S. Enoch, S. Guenneau, Biharmonic split ring resonator metamaterial: artificially dispersive effective density in thin periodically perforated plates, Europhys. Lett. 107 (4) (2014) 44002.

[40] H.-W. Dong, S.-D. Zhao, Y.-S. Wang, C. Zhang, Topology optimization of anisotropic broadband double-negative elastic metamaterials, J. Mech. Phys. Solids 105 (2017) 54-80.

[41] A. Krushynska, M. Miniaci, F. Bosia, N. Pugno, Coupling local resonance with bragg band gaps in single-phase mechanical metamaterials, Extreme Mech. Lett. 12 (2017) 30-36. 
[42] O. R. Bilal, A. Foehr, C. Daraio, Observation of trampoline phenomena in 3D-printed metamaterial plates, Extreme Mech. Lett. 15 (2017) 103-107.

[43] J. M. Kweun, H. J. Lee, J. H. Oh, H. M. Seung, Y. Y. Kim, Transmodal Fabry-Pérot resonance: theory and realization with elastic metamaterials, Phys. Rev. Lett. 118 (20) (2017) 205901.

[44] P. Gao, A. Climente, J. Sánchez-Dehesa, L. Wu, Theoretical study of platonic crystals with periodically structured $N$-beam resonators, J. Appl. Phys. 123 (9) (2018) 091707.

[45] H. Al Babaa, M. Attarzadeh, M. Nouh, Experimental evaluation of structural intensity in 2D plate-type locally resonant elastic metamaterials, J. Appl. Mech. https://doi. org/10.1115/1.4039042.

[46] A. Climente, P. Gao, L. Wu, J. Sánchez-Dehesa, Scattering of flexural waves from an $N$-beam resonator in a thin plate, J. Acoust. Soc. Am. 142 (5) (2017) 3205-3215.

[47] K. F. Graff, Wave Motion in Elastic Solids, Courier Corporation, New York, 2012.

[48] A. Climente, A. N. Norris, J. Sánchez-Dehesa, Scattering of flexural waves from a hole in a thin plate with an internal beam, J. Acoust. Soc. Am. 137 (1) (2015) 293-302.

[49] G. Huang, C. Sun, Band gaps in a multiresonator acoustic metamaterial, J. Vib. Acoust. 132 (3) (2010) 031003.

[50] Y. Xiao, J. Wen, X. Wen, Longitudinal wave band gaps in metamaterialbased elastic rods containing multi-degree-of-freedom resonators, New J. Phys. 14 (3) (2012) 033042.

[51] R. Zhu, X. Liu, G. Hu, C. Sun, G. Huang, A chiral elastic metamaterial beam for broadband vibration suppression, J. Sound Vib. 333 (10) (2014) $2759-2773$.

[52] M. V. Barnhart, X. Xu, Y. Chen, S. Zhang, J. Song, G. Huang, Experimental demonstration of a dissipative multi-resonator metamaterial for broadband elastic wave attenuation, J. Sound Vib. 438 (2019) 1-12. 


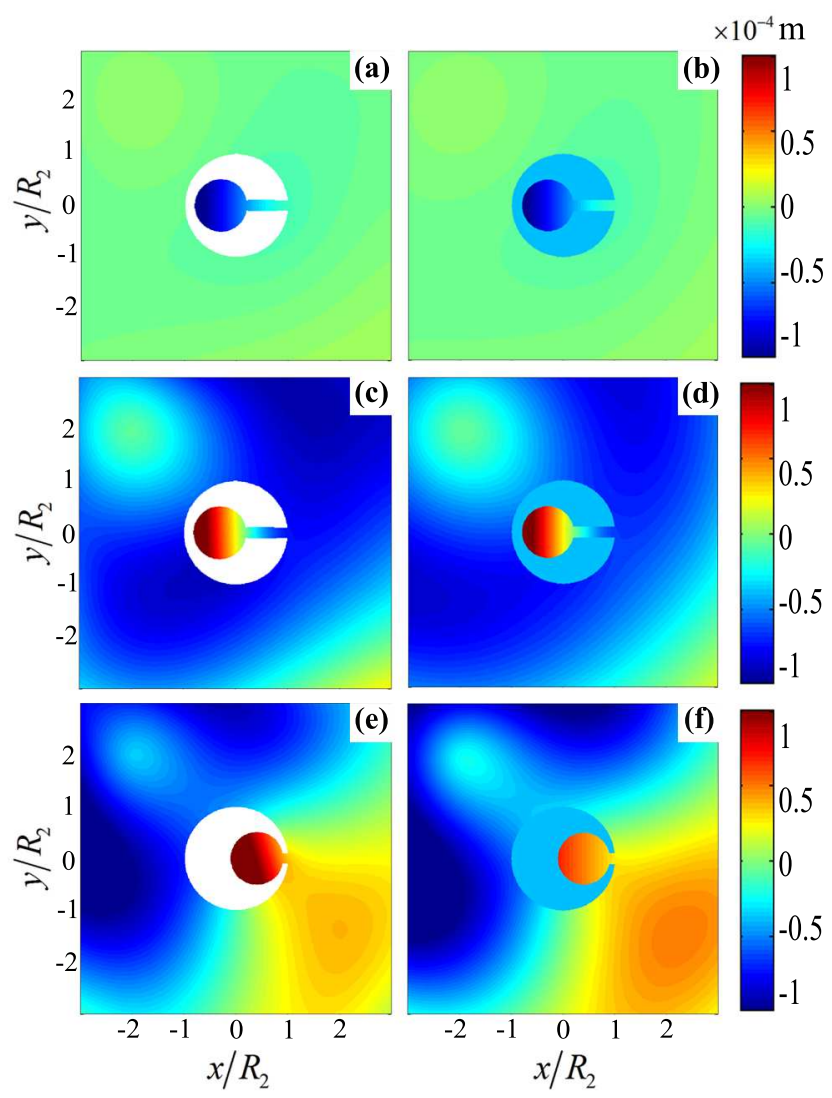

Fig. 2. (Color online) Snapshots of the out-of-plane displacements obtained from the scattering of a cylindrical wave with a resonant cavity containing a 1-beam resonator. The left and right panels represent results obtained from 3D full wave simulations [(a), (c) and (e)] and the 2D semi-analytical algorithm [(b), (d) and (f)], respectively. The resonators in the top [(a) and (b)] and middle panels [(c) and (d)] differ in the mass of the inner plate, while the resonators in the middle [(c) and (d)] and bottom panels [(e) and (f)] differ in the length of the rectangular beam. The cylindrical waves are excited by a punctual source placed at $(-2,2)$ with wavenumber $k_{p} R_{2}=0.20 \pi$ for the patterns shown in the top and middle panels, and $k_{p} R_{2}=0.26 \pi$ for the case shown in the bottom panels. 

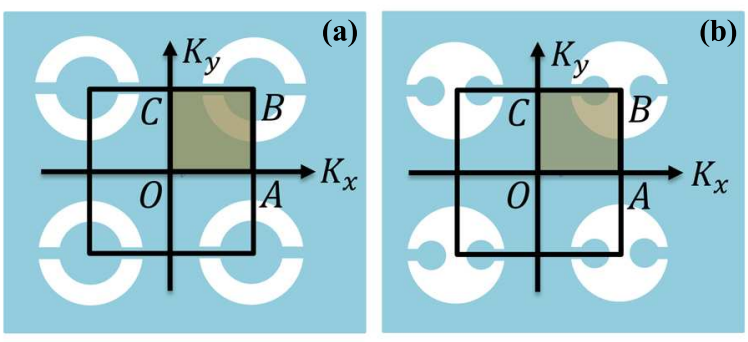

Fig. 3. (Color online) Schemes of the lattices in real space together with the definitions of the high symmetry directions in reciprocal space, where the irreducible Brillouin zones are depicted in gray. There is always one beam structured along the OA direction for each resonator in the square lattice. For illustration, only two beams are drawn for (a) the $N$-beam resonator and (b) the multiple 1-beam resonators. 


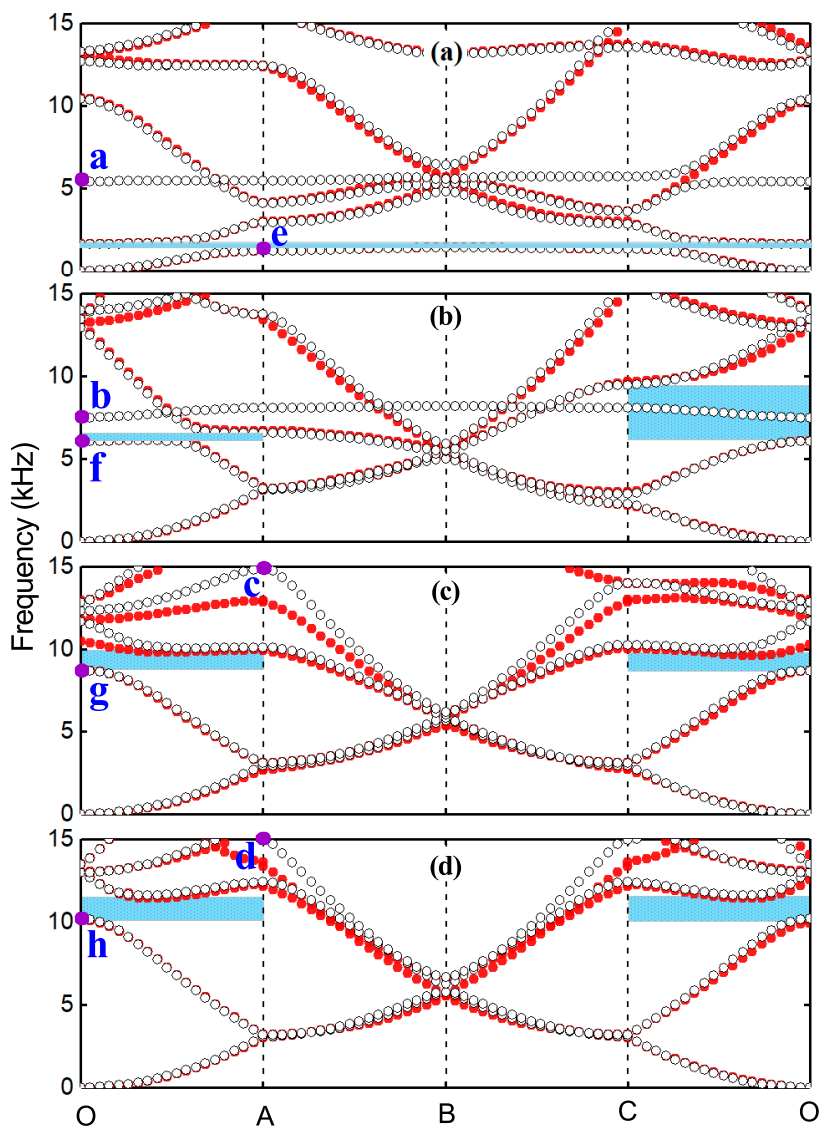

Fig. 4. (Color online) Band structures for lattices of $N$-beam resonators calculated with the commercial finite element package (hollow circles) and the multiple scattering algorithm (solid circles). From top to bottom panels, the colored regions bound the resonant bandgaps in lattices of (a) 1-, (b) 2-, (c) 3and (d) 4-beam resonators, respectively. Bands of interest are marked out at selected points a-h. 


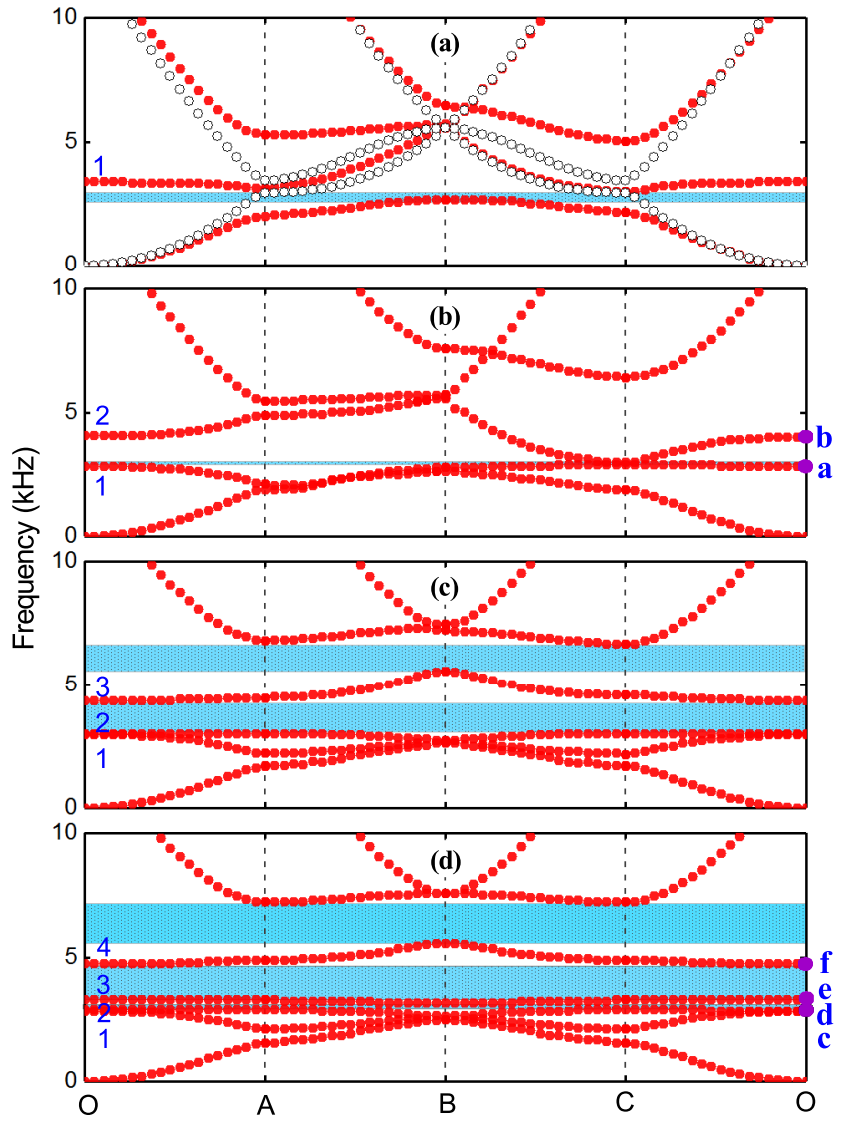

Fig. 5. (Color online) Flexural band structures (solid circles) for square lattices of cavities containing multiple $(M)$ 1-beam resonators. Results are shown for (a) $M=1$, (b) $M=2$, (c) $M=3$ and (d) $M=4$. The hollow circles in (a) represent the dispersion relation obtained for a lattice of cavities without resonators. Results are obtained by using a multiple scattering algorithm. The colored regions define the complete bandgaps. In each plot, the resonance induced bands are labeled with increasing numbers, and eigenmodes of interest are labeled from a to $\mathbf{f}$ at the origin of the reciprocal space. 


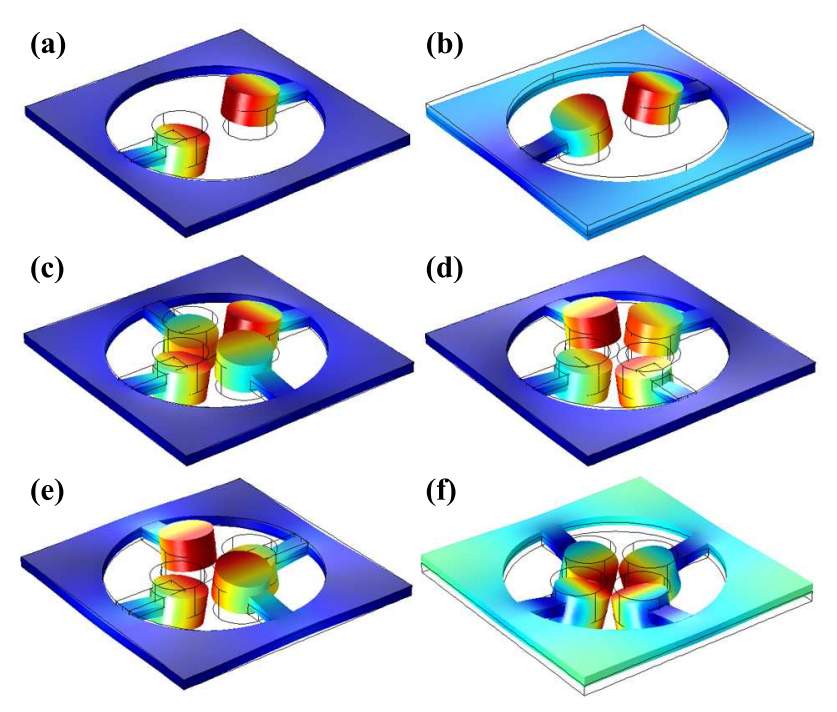

Fig. 6. (Color online) Eigenmodes calculated at the center of Brillouin zone. The figure numbers (a) to (f) correspond, respectively, to the points labeled from a to $\mathbf{f}$ in Fig. 5. 
(a)

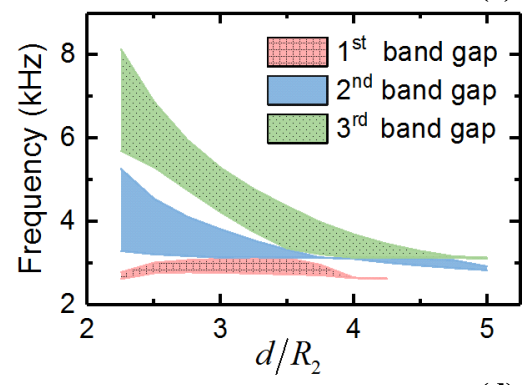

(d)

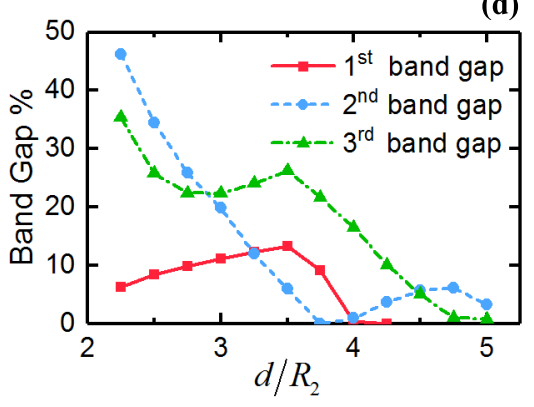

(b)

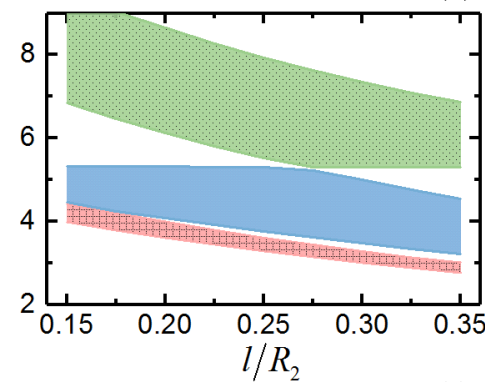

(e)

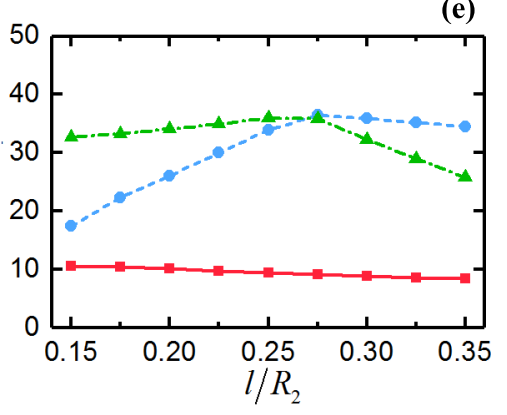

(c)

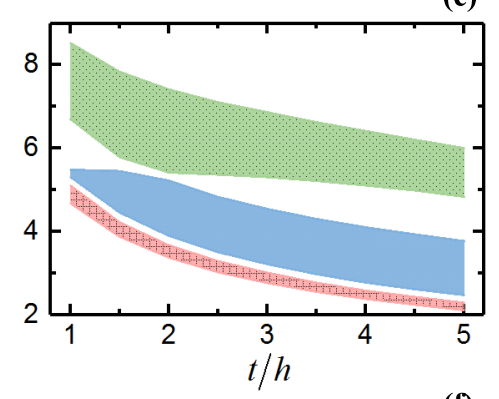

(f)

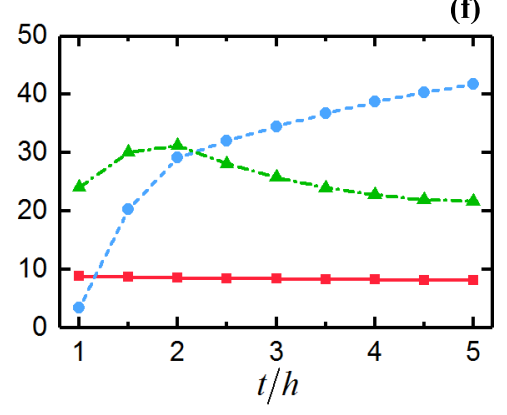

Fig. 7. (Color online) Parametric study on the resonant bandgaps for lattices of cavities containing four 1-beam resonators. Edges of the complete bandgaps are plotted as a function of (a) lattice period $d$, (b) beam length $\ell$ and (c) mass thickness $t$. Their normalized widths correspond to (d), (e) and (f), respectively. Except the variables, other parameter values used here are the same as those employed in the calculation of Fig. 5. 
(a)

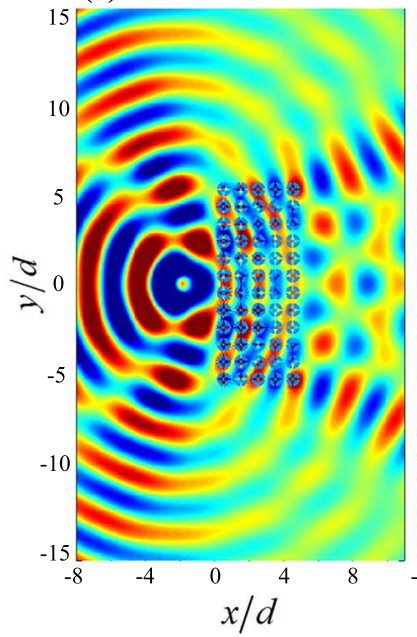

(b)

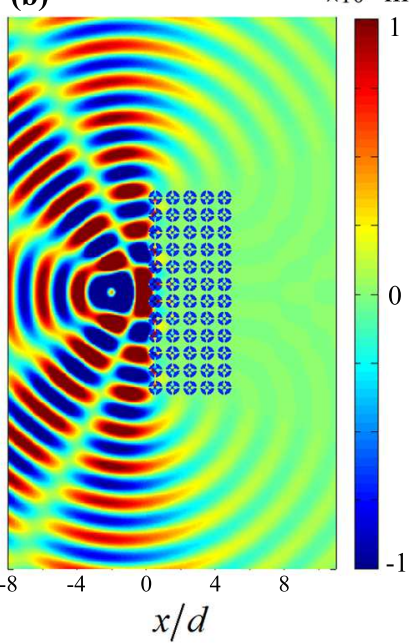

Fig. 8. (Color online) Snapshots of the out-of-plane displacements obtained when cylindrical waves interact with a metamaterial slab made of five rows of cavities containing four 1-beam resonators. Results are obtained in the framework of the multiple scattering method for a punctual sound source emitting waves with frequency (a) $1.8 \mathrm{kHz}$ and (b) $3.6 \mathrm{kHz}$. These frequencies fall in a partial and a complete bandgaps, respectively.

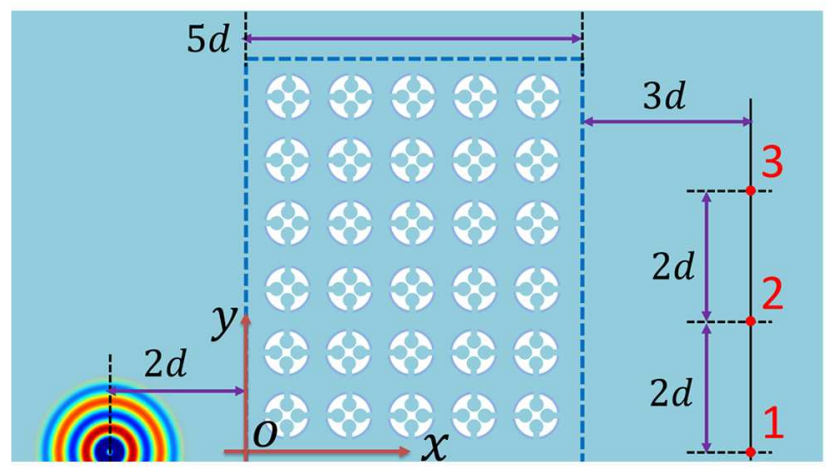

Fig. 9. (Color online) Schematic diagram of a platonic crystal slab consisting of $5 \times 12$ cavities containing four 1-beam resonators. For simplicity, only the upper half of the slab is depicted. A punctual source is placed in front of the slab to excite cylindrical waves. The vibration behind the slab is calculated at three different positions: 1,2 and 3, respectively. 

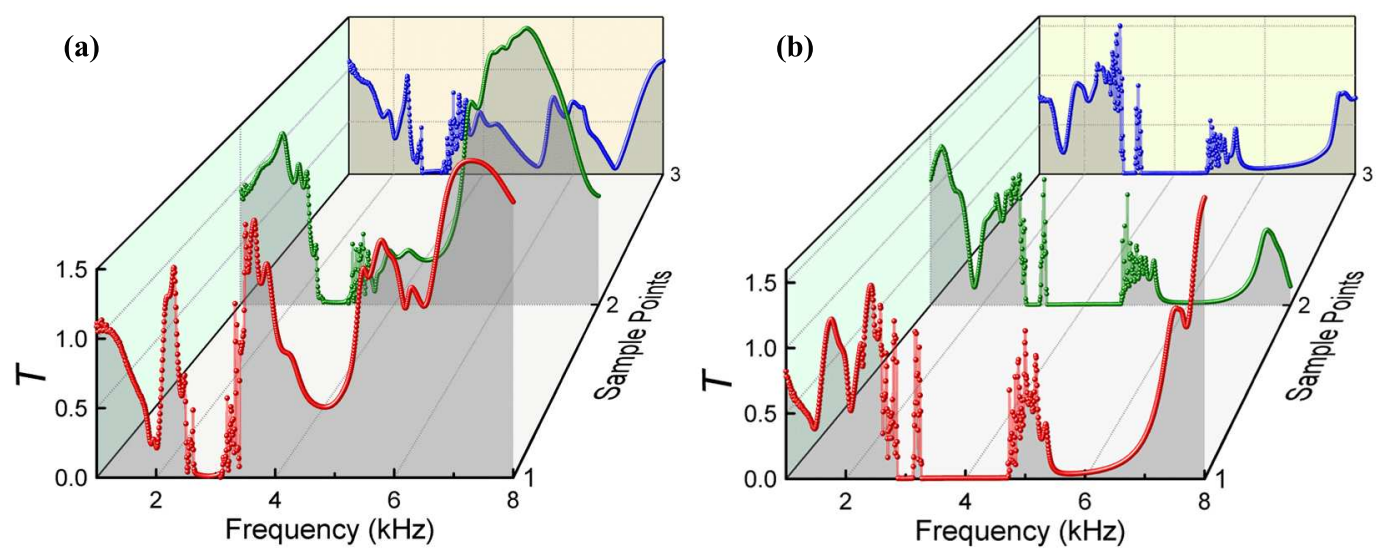

Fig. 10. (Color online) Vibration transmission for flexural waves passing through a metamaterial slab made of resonant cavities containing: (a) a single 1-beam resonator and (b) four 1-beam resonators. They are calculated at the three observation points described in Fig. 9. The valleys with zero transmission agree fairly well with the complete bandgaps predicted in Figs. 5(a) and $5(\mathrm{~d})$, respectively.

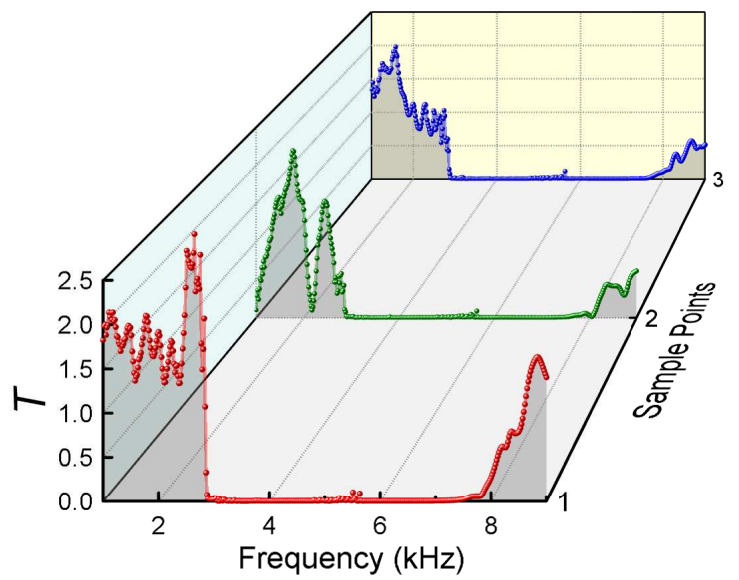

Fig. 11. (Color online) Vibration transmission for flexural waves passing through a composite slab composed of three metamaterial slabs made of resonant cavities differing in their beam length $\ell$. They are calculated at the three observation points described in Fig. 9. 

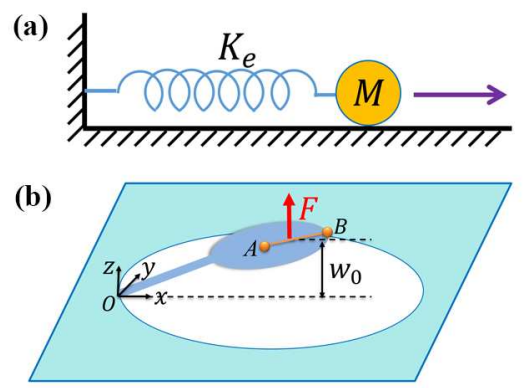

Fig. B.1. (color online) Analogous spring-mass oscillator representation of a 1-beam resonator. (a) Scheme of a simple spring-mass oscillator. (b) Scheme of a 1-beam resonator, its analogue in a thin plate. 


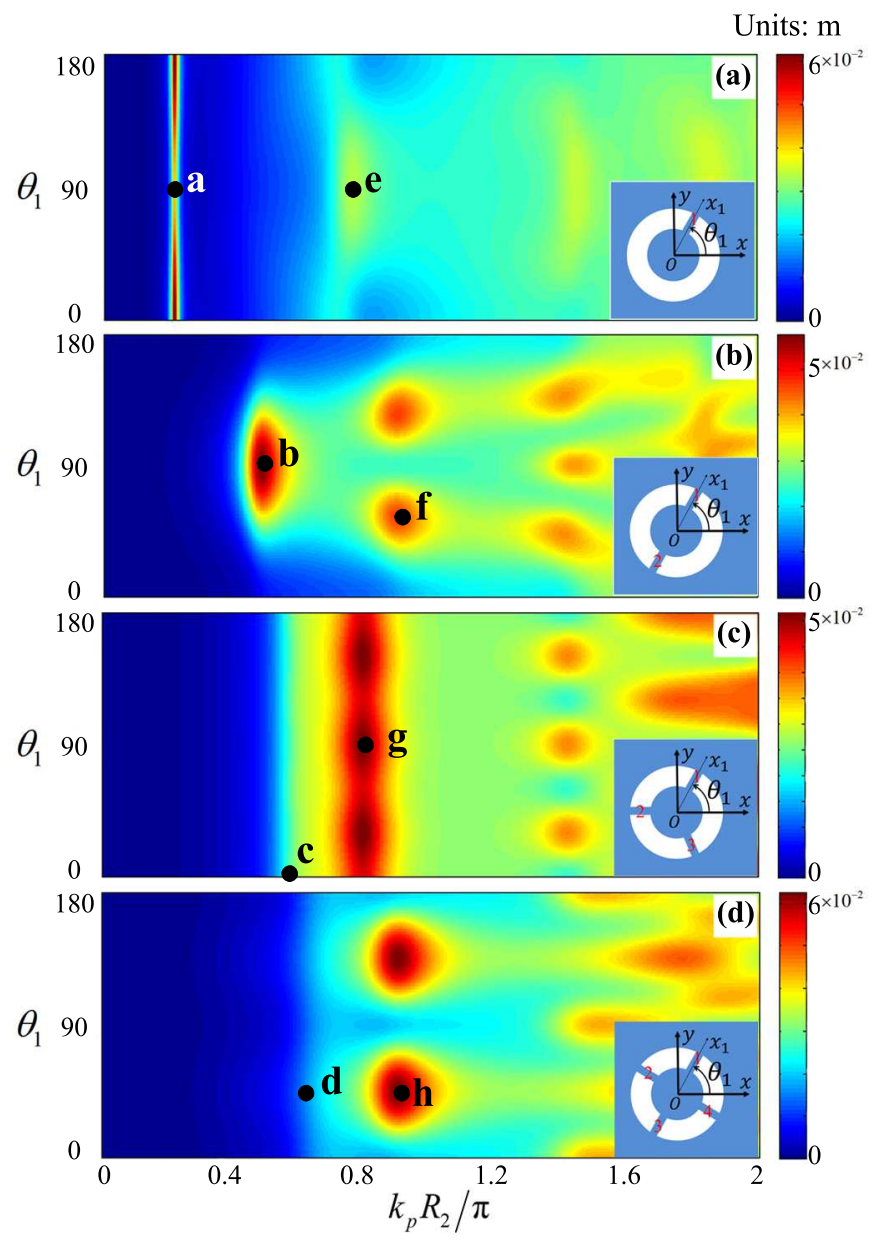

Fig. C.1. (Color online) Scattering cross-section for different $N$-beam resonators: (a) $N=1$, (b) $N=2$, (c) $N=3$ and (d) $N=4$. The results are obtained by considering the interaction of a plane wave propagating along the positive $x$-axis with the corresponding $N$-beam resonator. In each plot, the excitation frequency, $k_{p} R_{2} / \pi$, is given in reduced units. The vertical axis denotes the tilt angle (in degrees) of one particular beam, which is defined as the first, $\theta_{1}$ (see the insets). The 0 degree corresponds to the situation in which the first beam is aligned along the positive $x$-axis, while the other beams are uniformly distributed inside the cavity. Some regions of interest are marked out at points a-h. 

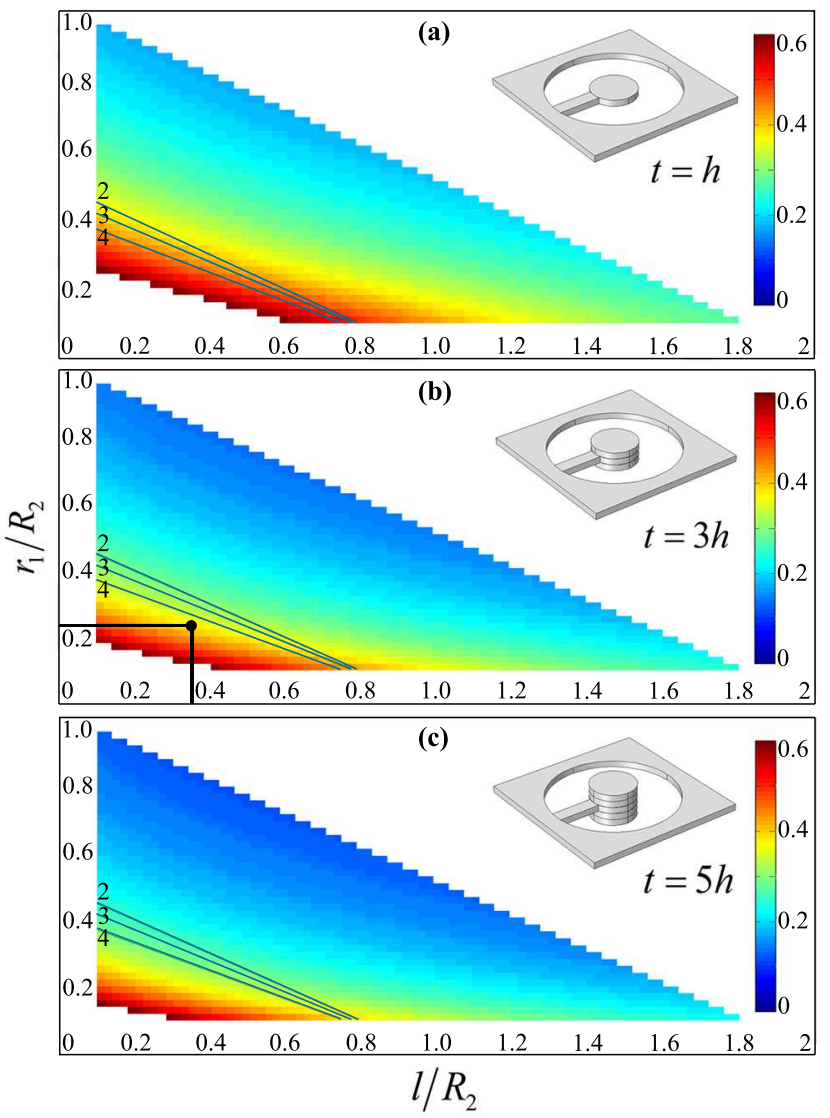

Fig. D.1. (Color online) Influence of the geometrical parameters on the frequency (in reduced units) of the fundamental resonance of a single 1-beam resonator. Results are obtained by extracting the lowest resonance in the maps of the scattering cross-section, as illustrated in Fig. C.1(a). From top to bottom panels, the thickness of the inner plate $(t)$ increases from (a) $h$ to (b) $3 h$ and (c) $5 h$. The colored regions define the available design spaces of the 1-beam resonator. Correspondingly, the lines marked with 2, 3 and 4 bound the design spaces of the multiple 1-beam resonators. 

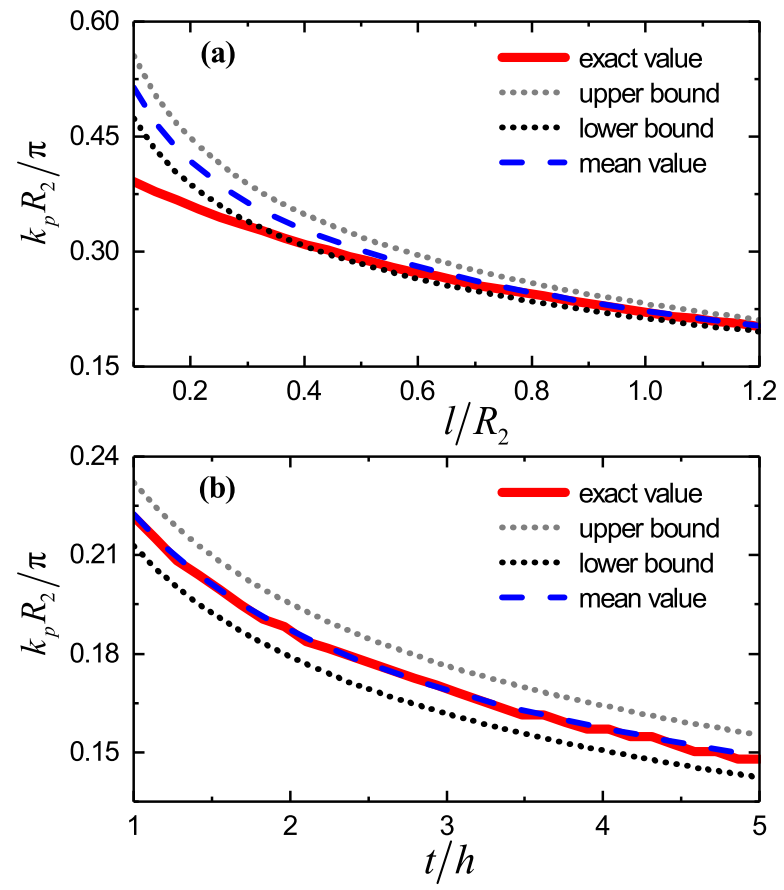

Fig. D.2. (Color online) Comparison between the resonant frequency in reduced units, $k_{p} R_{2} / \pi$, predicted by the semi-analytical model (solid lines) and the simple spring-mass model described in Appendix B (dashed and dotted lines). The dependence on $\ell$ and $t$ is reported in (a) and (b), respectively. 